\title{
Magnetic order, magnetic correlations, and spin dynamics in the pyrochlore antiferromagnet $\mathrm{Er}_{2} \mathbf{T i}_{2} \mathbf{O}_{7}$
}

\author{
P. Dalmas de Réotier, ${ }^{1}$ A. Yaouanc, ${ }^{1, *}$ Y. Chapuis,${ }^{1}$ S. H. Curnoe,${ }^{2}$ B. Grenier, ${ }^{1}$ E. Ressouche, ${ }^{1}$ C. Marin,,${ }^{1}$ J. Lago, ${ }^{3}$ \\ C. Baines, ${ }^{4}$ and S. R. Giblin ${ }^{5}$ \\ ${ }^{1}$ Institut Nanosciences et Cryogénie, SPSMS, CEA and Université Joseph Fourier, F-38054 Grenoble, France \\ ${ }^{2}$ Department of Physics and Physical Oceanography, Memorial University, St. John's, NL, Canada \\ ${ }^{3}$ Department of Inorganic Chemistry, Universidade del País Vasco, 48080 Bilbao, Spain \\ ${ }^{4}$ Laboratory for Muon-Spin Spectroscopy, Paul Scherrer Institute, 5232 Villigen-PSI, Switzerland \\ ${ }^{5}$ ISIS Facility, Rutherford Appleton Laboratory, Chilton, Didcot, OX11 0QX, UK
}

(Received 10 July 2012; revised manuscript received 31 August 2012; published 17 September 2012)

\begin{abstract}
$\mathrm{Er}_{2} \mathrm{Ti}_{2} \mathrm{O}_{7}$ is believed to be a realization of an $X Y$ antiferromagnet on a frustrated lattice of corner-sharing regular tetrahedra. It is presented as an example of the order-by-disorder mechanism in which fluctuations lift the degeneracy of the ground state, leading to an ordered state. Here we report detailed measurements of the low-temperature magnetic properties of $\mathrm{Er}_{2} \mathrm{Ti}_{2} \mathrm{O}_{7}$, which displays a second-order phase transition at $T_{\mathrm{N}} \simeq 1.2 \mathrm{~K}$ with coexisting short- and long-range orders. Magnetic susceptibility studies show that there is no spin-glass-like irreversible effect. Heat capacity measurements reveal that the paramagnetic critical exponent is typical of a 3-dimensional $X Y$ magnet while the low-temperature specific heat sets an upper limit on the possible spin-gap value and provides an estimate for the spin-wave velocity. Muon spin relaxation measurements show the presence of spin dynamics in the nanosecond time scale down to $21 \mathrm{mK}$. This time range is intermediate between the shorter time characterizing the spin dynamics in $\mathrm{Tb}_{2} \mathrm{Sn}_{2} \mathrm{O}_{7}$, which also displays long- and short-range magnetic order, and the time scale typical of conventional magnets. Hence the ground state is characterized by exotic spin dynamics. We determine the parameters of a symmetry-dictated Hamiltonian restricted to the spins in a tetrahedron, by fitting the paramagnetic diffuse neutron scattering intensity for two reciprocal lattice planes. These data are recorded in a temperature region where the assumption that the correlations are limited to nearest neighbors is fair.
\end{abstract}

DOI: 10.1103/PhysRevB.86.104424

PACS number(s): 75.40.-s, 75.10.Dg, 76.75.+i

\section{INTRODUCTION}

Because of the geometrical frustration of their magnetic superexchange interactions, the insulating pyrochlore compounds $R_{2} M_{2} \mathrm{O}_{7}$, where $R$ stands for a magnetic rare-earth ion and $M=\mathrm{Ti}$ or Sn, display a variety of unusual magnetic behaviors. ${ }^{1}$ Examples include (i) spin-ice systems $\mathrm{Ho}_{2} \mathrm{Ti}_{2} \mathrm{O}_{7}$ and $\mathrm{Dy}_{2} \mathrm{Ti}_{2} \mathrm{O}_{7}, 2,3$ (ii) $\mathrm{Yb}_{2} \mathrm{Ti}_{2} \mathrm{O}_{7}$ with a sharp transition in the spin dynamics fingerprinted by a pronounced peak in the specific heat, ${ }^{4}$ and (iii) $\mathrm{Tb}_{2} \mathrm{Sn}_{2} \mathrm{O}_{7}$ in which magnetic Bragg reflections are observed at low temperature by neutron diffraction, ${ }^{5}$ while no spontaneous magnetic field is found by the muon spin rotation $(\mu \mathrm{SR})$ technique. ${ }^{6}$ In addition, even when a spontaneous field and magnetic Bragg reflections are detected, as is expected for a conventional ordered magnet, persistent spin dynamics in the ordered state are surprisingly observed, e.g., in $\mathrm{Gd}_{2} \mathrm{Ti}_{2} \mathrm{O}_{7}$ and $\mathrm{Gd}_{2} \mathrm{Sn}_{2} \mathrm{O}_{7}{ }^{7-13}$ In terms of crystal-field anisotropy, the spin-ice systems are strongly Ising-like. $\mathrm{Tb}_{2} \mathrm{Sn}_{2} \mathrm{O}_{7}$ has also an Ising anisotropy, but not so strong. $\mathrm{Yb}_{2} \mathrm{Ti}_{2} \mathrm{O}_{7}$ is $X Y$-like from the crystal-field point of view and the Gd compounds are approximately isotropic.

Although $\mathrm{Yb}_{2} \mathrm{Ti}_{2} \mathrm{O}_{7}$ is $X Y$-like, its magnetic moments are not perpendicular to the local $\langle 111\rangle$ axes and it does not display long-range magnetic order, ${ }^{4,14}$ although this absence of order has been disputed ${ }^{15,16}$ and is still under debate. ${ }^{17}$ These anisotropy and absence of long-range order also pertain for $\mathrm{Yb}_{2} \mathrm{GaSbO}_{7}{ }^{18}$ Hence, it was of great interest when $\mathrm{Er}_{2} \mathrm{Ti}_{2} \mathrm{O}_{7}$ was reported to be $X Y$-like and to display long-range order at low temperature with the $\mathrm{Er}^{3+}$ magnetic moments perpendicular to their local [111] axes. ${ }^{19}$ Later on, however, coexisting short- and long-range orders were found and soft collective modes were detected. ${ }^{20}$ The presence of the soft modes has been attributed to the incommensurate value of the canting angle. ${ }^{21}$ These astonishing inferences call for more detailed data and analysis. This is the purpose of this work. One of the experimental advantages of $\mathrm{Er}_{2} \mathrm{Ti}_{2} \mathrm{O}_{7}$ over $\mathrm{Yb}_{2} \mathrm{Ti}_{2} \mathrm{O}_{7}$ and $\mathrm{Yb}_{2} \mathrm{GaSbO}_{7}$ is the possibility to produce large high-quality crystals.

Another reason for the interest in the $\mathrm{Er}_{2} \mathrm{Ti}_{2} \mathrm{O}_{7}$ system is the following. As a realization of an $X Y$ antiferromagnet on a pyrochlore lattice, it is a natural candidate for observing the phenomenon of order by disorder which has been discussed theoretically for more than three decades since the pioneering study by Villain and coworkers. ${ }^{22}$ Bramwell et al. indeed showed that while the zero-temperature ground state is degenerate, thermal fluctuations select a subset of the manifold and induce a first-order phase transition to a conventional Néel ground state. ${ }^{23}$ The order-by-disorder mechanism has been confirmed in several subsequent works, see, e.g., Refs. 19,24-26, and interestingly the more recent studies consider the effect of quantum fluctuations and tend to explain the second-order nature of the transition experimentally observed in $\mathrm{Er}_{2} \mathrm{Ti}_{2} \mathrm{O}_{7}$.

The organization of this paper is as follows. Section II gives a survey of the physical properties of $\mathrm{Er}_{2} \mathrm{Ti}_{2} \mathrm{O}_{7}$ and discusses its magnetic structure. In Sec. III we describe the growth of the single crystals, their basic characterizations, and the experimental methods used in the present work. Section IV presents our investigation of the bulk properties 
of the system, including magnetic-susceptibility and specificheat measurements and their analysis. The following section (Sec. V) deals with the microscopic techniques, i.e., muon spin relaxation and neutron scattering in the paramagnetic phase. A summary of our key results is given in Sec. VI. The physics of effective one-half spins (Kramers doublets) on a tetrahedron is described in Appendix A. Appendix B outlines the calculation needed for the analysis of the specific-heat data presented in Sec. IV.

\section{PHYSICAL PROPERTIES OF $\mathrm{Er}_{2} \mathrm{Ti}_{2} \mathrm{O}_{7} \mathrm{AND}$ MAGNETIC STRUCTURE}

Erbium titanate, $\mathrm{Er}_{2} \mathrm{Ti}_{2} \mathrm{O}_{7}$, is an insulating pyrochlore compound that crystallizes into the cubic space group $F d \overline{3} \mathrm{~m}$, with the lattice parameter $a=10.0727$ (1) $\AA$ at room temperature and $x=0.3278(8)$, the free position parameter allowed by the space group which characterizes the $48 \mathrm{f}$ site occupied by oxygen. $^{27}$

The $\mathrm{Er}^{3+}$ ions, which occupy the $16 \mathrm{~d}$ Wyckoff positions in the space group, are located at the vertices of a corner-sharing network of tetrahedra; see Fig. 1. A single tetrahedron with four Er sites comprises the primitive unit cell. $\mathrm{The}^{3+} \mathrm{Fr}^{3+}$ crystal sites are all equivalent and the local symmetry is $D_{3 d}$, where the 3-fold axes pass through the center of a tetrahedron, in the directions [111], [ $\overline{1} \overline{1} 1],[\overline{1} 1 \overline{1}]$, and [1 $\overline{1} \overline{1}]$ for the four sites numbered 1, 2, 3, and 4, respectively, on a single tetrahedron. According to Hund's rules, the total angular momentum of the $\mathrm{Er}^{3+}$ ion in its ground multiplet is $J=15 / 2$. The $16-$ fold degeneracy is lifted into Kramers doublets by the crystal electric field (CEF).

The ground-state doublet can be described as an effective spin $S=1 / 2$ with a quantization axis $z$ parallel to the local trigonal axis. It is well isolated from the excited doublets, the lowest being at about $74 \mathrm{~K}$ above the ground state in temperature units. ${ }^{19}$ We shall write the CEF ground-state

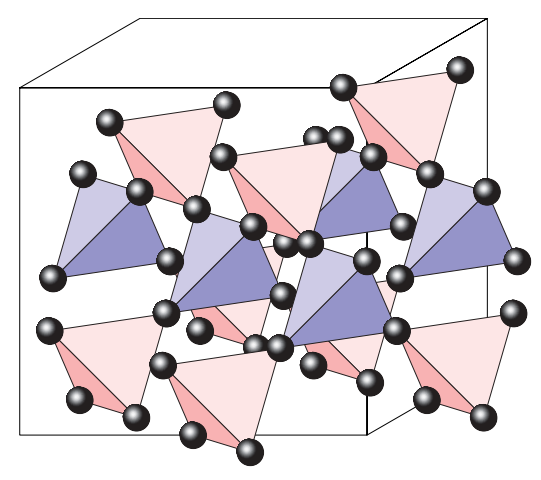

FIG. 1. (Color online) The network of corner-sharing regular tetrahedra formed by the rare-earth atoms in the pyrochlore structure in which $\mathrm{Er}_{2} \mathrm{Ti}_{2} \mathrm{O}_{7}$ crystallizes. The axis of trigonal symmetry at the position of a rare earth is one of the cube diagonals. There are two types of tetrahedra in the network, which differ by their orientation: Type B is rotated by $90^{\circ}$ about the cubic axes with respect to type A. We distinguish the two sets by two colors in the drawing. Since each rare earth is at a corner shared by two tetrahedra, one of each kind, either the set of the four corners of all the A tetrahedra or the set of the four corners of all the B tetrahedra is sufficient to describe the $\mathrm{Er}^{3+}$ lattice. doublet wave functions as $\left|\phi_{0}^{ \pm}\right\rangle$. This doublet is characterized by its spectroscopic factors along and perpendicular to the trigonal axis, $g_{\|}$and $g_{\perp}$, respectively. From a global analysis of the CEF for the pyrochlore $R_{2} \mathrm{Ti}_{2} \mathrm{O}_{7}$ series it has been deduced that $g_{\|}=2 g_{J}\left|\left\langle\phi_{0}^{ \pm}\left|J_{z}\right| \phi_{0}^{ \pm}\right\rangle\right|=1.8(5)$ and $g_{\perp}=$ $g_{J}\left|\left\langle\phi_{0}^{ \pm}\left|J_{ \pm}\right| \phi_{0}^{\mp}\right\rangle\right|=7.7(1) .{ }^{28}$ Here $g_{J}=6 / 5$ is the Landé factor. These spectroscopic factors are related to matrix elements that we shall need for the analysis of neutron scattering data. We have

$$
\begin{gathered}
j_{\mathrm{CEF}} \equiv\left\langle\phi_{0}^{+}\left|J_{z}\right| \phi_{0}^{+}\right\rangle=-\left\langle\phi_{0}^{-}\left|J_{z}\right| \phi_{0}^{-}\right\rangle=0.75(20), \\
t_{\mathrm{CEF}} \equiv\left\langle\phi_{0}^{ \pm}\left|J_{ \pm}\right| \phi_{0}^{\mp}\right\rangle=6.42(8),
\end{gathered}
$$

where $J_{ \pm} \equiv J_{x} \pm i J_{y}$. The matrix elements obviously refer to quantities written in local axes; see Appendix A 1 for a discussion. By definition, the large difference between $j_{\mathrm{CEF}}$ and $t_{\mathrm{CEF}}$ (and obviously also between $g_{\|}$and $g_{\perp}$ ) reflects the strong CEF anisotropy of the $X Y$ type of the Er spins (in contrast to the Ising limit for which $\left.j_{\mathrm{CEF}} \gg t_{\mathrm{CEF}}\right)$.

The compound displays a magnetic phase transition at $T_{\mathrm{N}} \simeq 1.2 \mathrm{~K}^{29}$ The large negative value of the Curie-Weiss temperature $\theta_{\mathrm{CW}}\left(\theta_{\mathrm{CW}}=-22 \mathrm{~K}\right.$ is deduced from susceptibility data measured between 20 and $50 \mathrm{~K}$; see Refs. 29 and 30) suggests a strong antiferromagnet coupling.

Neutron diffraction shows the magnetic structure below $T_{\mathrm{N}}$ to be noncollinear with the propagation vector $\mathbf{k}=(0,0,0)$. ${ }^{19}$ From polarized neutron diffraction, the $\mathrm{Er}^{3+}$ magnetic moment is determined to be $m=3.25(9) \mu_{\mathrm{B}}$ at low temperature. ${ }^{31}$ A note of caution seems justified at this juncture: $m$ is not directly related to the spectroscopic factors which have been determined for a paramagnetic ion, since the molecular field has to be taken into account for an estimation of $m$. The diffraction data have been originally described ${ }^{19,31}$ with the $\Gamma_{3}^{+}$irreducible representation. ${ }^{32}$ We notice that this description also considered in Refs. 20,33, and 34 has been recently disputed by Briffa et al. ${ }^{21}$

In fact the available microscopic information provides an insight into the moment orientation. Let us consider a one-half spin subjected to a molecular field oriented at a polar angle $\theta$ from the [111] axis. The following relation can be derived: ${ }^{35,36}$

$$
\tan \theta=\frac{g_{\|}}{g_{\perp}} \sqrt{\frac{\mu_{\mathrm{B}}^{2} g_{\|}^{2}-4 m^{2}}{4 m^{2}-\mu_{\mathrm{B}}^{2} g_{\perp}^{2}}} .
$$

Numerically this gives $\theta \approx 20^{\circ}$. This means that the field is not far from being parallel to the [111] axis. However, the polar angle $\phi$ of $\mathbf{m}$ is given by $\tan \phi=\left(g_{\perp} / g_{\|}\right)^{2} \tan \theta$; i.e., $\phi \approx 80^{\circ}$. Taking into account the uncertainties on $g_{\perp}, g_{\|}$, and $m$, this analysis indicates that the moment is perpendicular to the local [111] axis, or at least close to being perpendicular. This is consistent with the magnetic structure first proposed by Champion et al., ${ }^{19}$ and invalidates the proposal of Ref. 21 .

Regardless of the magnetic structure, the $(2,2,0)$ Bragg reflection has been shown to be anomalous with broad tails. ${ }^{20}$ Quantitatively, we find that the intensity around this position is proportional to $\left|q-q_{(2,2,0)}\right|^{-\eta}$ with $\eta \simeq 0.6$ for $\left|q-q_{(2,2,0)}\right|>$ $0.03 \AA^{-1}$; see Fig. 2. The value of the exponent $\eta$ is much reduced compared to $\eta \simeq 1.8$ found in $\mathrm{Tb}_{2} \mathrm{Sn}_{2} \mathrm{O}_{7}{ }^{6,37}$ For a conventional ordered compound the neutron intensity would be Gaussian-like, i.e., with a much steeper slope. 


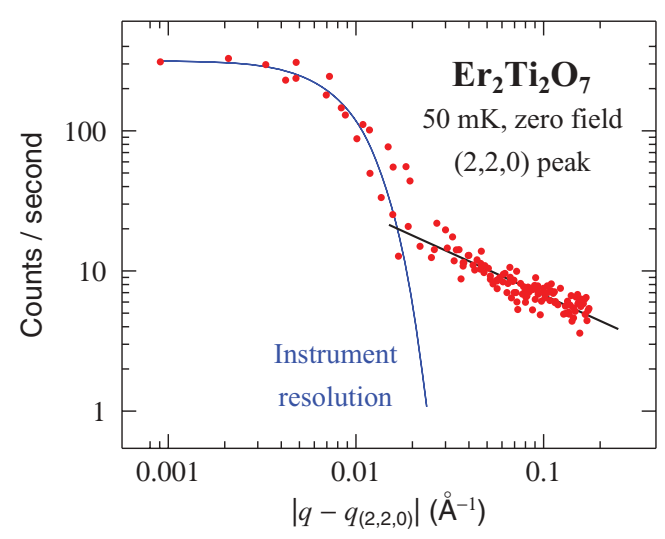

FIG. 2. (Color online) Neutron intensity for the $(2,2,0)$ Bragg peak of $\mathrm{Er}_{2} \mathrm{Ti}_{2} \mathrm{O}_{7}$ plotted as a function of $\left|q-q_{(2,2,0)}\right|$. The data are from Ruff et al. (Ref. 20). The instrumental resolution is determined from the reflection measured under a $3 \mathrm{~T}$ magnetic field. The line for $\left|q-q_{(2,2,0)}\right| \geqslant 0.015 \AA^{-1}$ is described in the main text.

Inelastic neutron scattering data recorded in zero field suggest the presence of magnetic soft modes, ${ }^{20}$ in agreement with the power-law behavior of the specific heat below $T_{\mathrm{N}}{ }^{34}$ At first sight this is surprising given the expected strong crystal-field anisotropy of the $\mathrm{Er}^{3+}$ ions. $^{21}$

\section{EXPERIMENTAL}

$\mathrm{Er}_{2} \mathrm{Ti}_{2} \mathrm{O}_{7}$ single crystals were grown by the floating zone technique using a commercial optical furnace. Feed rods were prepared from high-purity oxides $\left(\mathrm{TiO}_{2}, 99.995 \%\right.$ and $\mathrm{Er}_{2} \mathrm{O}_{3}$, $99.99 \%$ ), mixed and heat treated up to $1180^{\circ} \mathrm{C}$. After sintering, a rod was heat treated up to $1350^{\circ} \mathrm{C}$ in air. Crystal growth conditions were optimized under air $(1 \ell / \mathrm{min})$ at the growth rate of $2 \mathrm{~mm} / \mathrm{h}$ coupled with a rotation rate of 30 rounds per minute. As with most of the titanate pyrochlores, $\mathrm{Er}_{2} \mathrm{Ti}_{2} \mathrm{O}_{7}$ crystallized rods are mostly transparent, with a slight pink color. No phases other than the cubic one with $F d \overline{3} m$ space group were detected by x-ray powder diffraction experiments; see Fig. 3 for an example. As-grown and postgrowth heat-treated crystals were characterized by specific-heat measurements. No noticeable

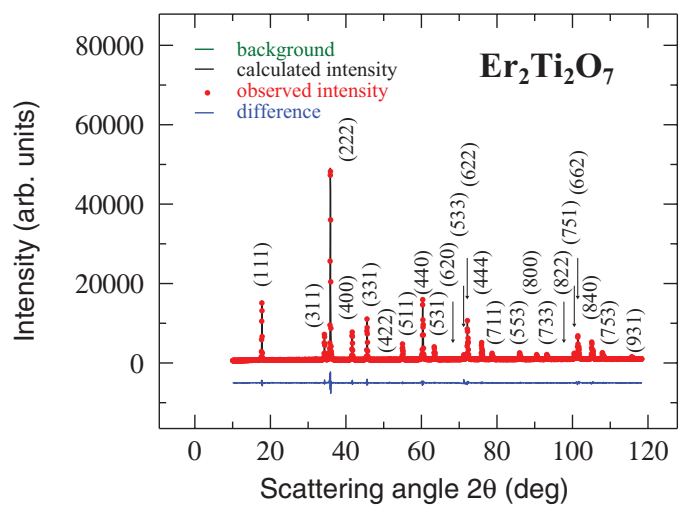

FIG. 3. (Color online) An example of an x-ray diffraction pattern recorded at room temperature for an $\mathrm{Er}_{2} \mathrm{Ti}_{2} \mathrm{O}_{7}$ powder obtained after crushing part of a crystal. The radiation used is Co $\mathrm{K}_{\alpha}$. The line at the bottom of the graph shows the difference between the data and the refinement model.

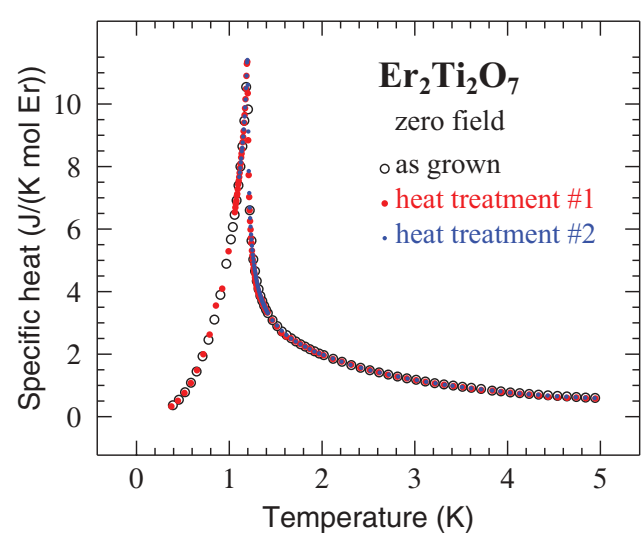

FIG. 4. (Color online) Specific heat versus temperature for three samples cut from an $\mathrm{Er}_{2} \mathrm{Ti}_{2} \mathrm{O}_{7}$ crystal prepared as indicated in the main text. For the first sample, no subsequent treatment has been performed, while the other two result from postgrowth heat treatments as follows. Case 1: Heat treatment for 7 days under oxygen at $1150^{\circ} \mathrm{C}$, followed by a slow cooling down to $400{ }^{\circ} \mathrm{C}$. Case 2 : Heat treatment for 7 days under argon at $1150{ }^{\circ} \mathrm{C}$, followed by a slow cooling down to $400{ }^{\circ} \mathrm{C}$.

differences were detected; see Fig. 4. This is in contrast to the case of $\mathrm{Tb}_{2} \mathrm{Ti}_{2} \mathrm{O}_{7}{ }^{38}$ The position of the peak provides a measure of the critical temperature. We get $T_{\mathrm{N}}=1.23(1)$ $\mathrm{K}$. For comparison the following values have already been published: 1.25 K (Ref. 29) and 1.173(2) K (Ref. 19) in reasonable agreement.

The investigation of the macroscopic properties of the system consisted of magnetic susceptibility experiments performed with a commercial magnetometer (magnetic property measurement system, Quantum Design, Inc.) down to $2 \mathrm{~K}$, and of heat capacity measurements. For this latter physical property, the temperature range from 0.48 to $20 \mathrm{~K}$ was investigated with a commercial calorimeter [physical property measurement system, (PPMS), Quantum Design, Inc.] equipped with a ${ }^{3} \mathrm{He}$ stage using a standard thermal relaxation method. Additional measurements between 0.11 and $2.50 \mathrm{~K}$ were performed with a homemade calorimeter inserted in a dilution refrigerator using a semiadiabatic technique.

The $\mu$ SR measurements were carried out at the European Muon Spectrometer of the ISIS facility (Rutherford Appleton Laboratory, United Kingdom) and the Low Temperature Facility of the Swiss Muon Source $(\mathrm{S} \mu \mathrm{S}$, Paul Scherrer Institute, Switzerland). The muon beam is pulsed at the former facility and pseudocontinuous at the latter.

The neutron scattering experiments were performed at the Institut Laue Langevin (ILL, Grenoble) with the liftingcounter diffractometer D23 of the CEA collaborating Research Group (CRG).

\section{BULK MEASUREMENTS}

Here we shall first discuss magnetic susceptibility measurements. Then we shall present zero-field specific-heat results and finish with the determination of the magnetic phase diagram using specific-heat data recorded under magnetic fields. 


\section{A. Magnetic susceptibility}

Since the magnetization measurements were performed on a needle-shaped sample and the field was applied along its long axis, the demagnetization field is negligible. Classically, the static magnetic susceptibility $\chi$ is expected to follow a Curie-Weiss law far from the ordering temperature in the paramagnetic regime. It reads

$$
\chi=\frac{C}{T-\theta_{\mathrm{CW}}},
$$

where the Curie constant $C$ can be expressed in terms of the so-called paramagnetic moment $m_{\text {para }}$ :

$$
C=\frac{1}{v} \frac{\mu_{0} m_{\mathrm{para}}^{2}}{3 k_{\mathrm{B}}}
$$

where $v=a^{3} / N_{\text {cell }}$ with $N_{\text {cell }}$ being the number of $\mathrm{Er}^{3+}$ ions in the cubic cell $\left(N_{\text {cell }}=16\right)$. For an isolated $\mathrm{Er}^{3+}$ ion, $m_{\text {para }}=$ $g_{J} \sqrt{J(J+1)} \mu_{\mathrm{B}}=9.58 \mu_{\mathrm{B}}$.

In Fig. 5 we display our result for the inverse of the static susceptibility versus temperature in a large temperature range. The Curie-Weiss law provides a good description of our data above $30 \mathrm{~K}$. The fit gives for the Curie-Weiss temperature $\theta_{\mathrm{CW}}=-17.5$ (3) $\mathrm{K}$ and $C=3.73$ (4) K. This means that $m_{\text {para }}=9.55(10) \mu_{\mathrm{B}}$, in agreement with the result for an isolated $\mathrm{Er}^{3+}$ ion. Because $\theta_{\mathrm{CW}}$ is negative, the dominant exchange interactions are antiferromagnetic. For comparison, fitting data recorded between 20 and $50 \mathrm{~K}$ on a powder sample in a field of $1 \mathrm{mT}$, Bramwell et al. ${ }^{30}$ found values of $\theta_{\mathrm{CW}}=-22.3(3) \mathrm{K}$ and $m_{\text {para }}=9.34(9) \mu_{\mathrm{B}}$. For data recorded between 50 and $300 \mathrm{~K}$ in a field of $50 \mathrm{mT}$ these authors obtain $\theta_{\mathrm{CW}}=-15.93(3) \mathrm{K}$ and $m_{\text {para }}=8.936(4) \mu_{\mathrm{B}}$, the latter at variance with the result for an isolated $\mathrm{Er}^{3+}$ ion. From our result, we compute for the frustration index ${ }^{39} f \equiv\left|\theta_{\mathrm{CW}}\right| / T_{\mathrm{N}}=$ 14. Since $f \gg 1, \mathrm{Er}_{2} \mathrm{Ti}_{2} \mathrm{O}_{7}$ is a strongly frustrated magnet. In addition, assuming the $\mathrm{Er}^{3+}$ magnetic moments to interact through a simple nearest-neighbor Heisenberg interaction with

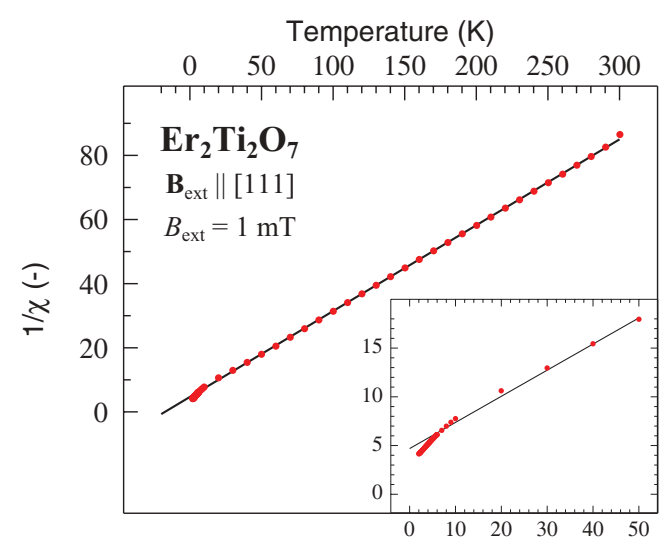

FIG. 5. (Color online) Inverse of the magnetic susceptibility of an $\mathrm{Er}_{2} \mathrm{Ti}_{2} \mathrm{O}_{7}$ crystal versus temperature in a large temperature range. The solid line results from a fit with the Curie-Weiss law. A field of $1 \mathrm{mT}$ is applied along a diagonal of the cubic crystal structure. The inset shows the low-temperature range of the data.

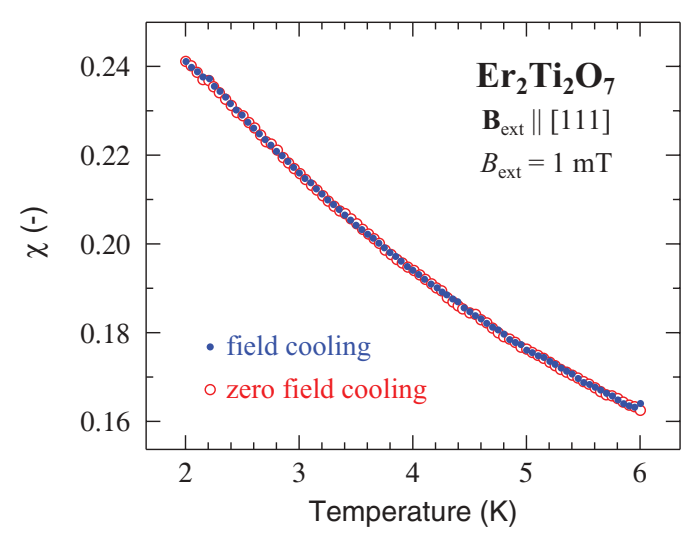

FIG. 6. (Color online) The magnetic susceptibility of an $\mathrm{Er}_{2} \mathrm{Ti}_{2} \mathrm{O}_{7}$ crystal versus temperature in the low-temperature region. The same magnetic response is observed using either the field- or zero-fieldcooling procedure, $\mathrm{fc}$ and zfc, respectively. A field of $1 \mathrm{mT}$ is applied along a diagonal of the cubic crystal structure. In the zfc mode the sample was warmed to $300 \mathrm{~K}$ and then cooled to $2 \mathrm{~K}$ before applying the magnetic field. In the fc mode the field was applied at $300 \mathrm{~K}$ and the sample was subsequently cooled down.

exchange integral $\mathcal{I}(\mathcal{I}>0)$, i.e.,

$$
\mathcal{H}=\frac{\mathcal{I}}{2} \sum_{i, j, i \neq j} \mathbf{J}_{i} \cdot \mathbf{J}_{j}=\mathcal{I} \sum_{\langle i, j\rangle} \mathbf{J}_{i} \cdot \mathbf{J}_{j},
$$

the molecular-field approximation predicts

$$
\mathcal{I}=\frac{3 k_{\mathrm{B}}\left|\theta_{\mathrm{CW}}\right|}{z_{\mathrm{nn}} J(J+1)} .
$$

We denote as $z_{\mathrm{nn}}$ the number of nearest neighbor $\mathrm{Er}^{3+}$ ions to a given $\mathrm{Er}^{3+}$ ion. In our case $z_{\mathrm{nn}}=6$. From the measured $\theta_{\mathrm{CW}}$ value and taking into account that $J=15 / 2$, we compute $\mathcal{I} / k_{\mathrm{B}}=0.138(2) \mathrm{K}$.

We have also measured the susceptibility for $2.0<T<$ $6.0 \mathrm{~K}$ under a field of $1 \mathrm{mT}$ applied along a [111] axis using two protocols; see Fig. 6. Contrary to a previous report, ${ }^{30}$ we do not observe any history-dependent effect at $T \leqslant 3.2 \mathrm{~K}$. Hence, there is no spin-glass-like irreversible effect for our $\mathrm{Er}_{2} \mathrm{Ti}_{2} \mathrm{O}_{7}$ crystals.

\section{B. Specific heat in zero magnetic field}

Here we present and discuss zero-field specific-heat data recorded for $\mathrm{Er}_{2} \mathrm{Ti}_{2} \mathrm{O}_{7}$ crystals. It is well known that they may lead to a characterization of the low-energy magnetic modes, detect indirectly a dynamical magnetic component in the ordered state, determine the universality class of the system under study, and gauge a possible residual entropy at low temperature.

In Fig. 7 we display our data, in the low-temperature regime.

While our results are in reasonable agreement with data published by Siddharthan et al., ${ }^{40}$ they differ drastically at low temperature with the ones of Blöte et al. ${ }^{29} \mathrm{We}$ do not understand the origin of the large difference with the data of Blöte et al. In the following we shall focus on the analysis of our data and the ones of Siddharthan et al. Note that here we have not considered the results of Sosin et al. ${ }^{34}$ since they do not extend to very low temperature. 


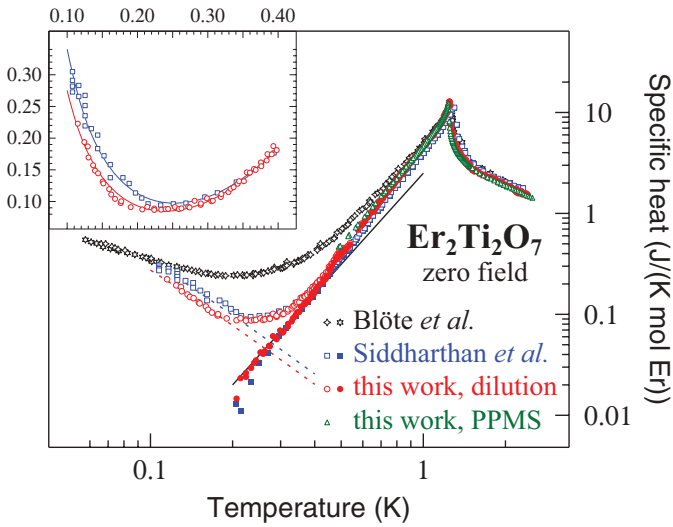

FIG. 7. (Color online) Low-temperature specific heat of $\mathrm{Er}_{2} \mathrm{Ti}_{2} \mathrm{O}_{7}$. The open symbols show our experimental data compared to literature results from Blöte et al. (Ref. 29) and Siddharthan et al. (Ref. 40). Note the large overlap between our results obtained with the PPMS and the homemade calorimeter inserted in a dilution refrigerator. The filled symbols present the electronic specific heat deduced from the data of Siddharthan et al. and this work. The dashed lines show the contributions from the nuclear specific heat and the full line results from a fit of the low-temperature electronic specific heat to the $C_{\text {elec }}=\mathcal{B} T^{3}$ law, with $\mathcal{B}=2.50$ (15) $\mathrm{J} \mathrm{K}^{-4} \mathrm{~mol}^{-1}$. The inset displays the very low temperature details. The solid lines result from fits as explained in the main text.

In the low-temperature range shown in Fig. 7 the specific heat arises from three origins: the contribution from the nuclei with nonzero spins at low temperature, then the lowenergy magnon modes at higher temperature and the critical fluctuations around $T_{\mathrm{N}}$. As a start we shall focus on the first two contributions, i.e., the specific heat of nuclear and magnon origins, $C_{\mathrm{n}}$ and $C_{\mathrm{sw}}$, respectively.

We begin with providing a theoretical background for these contributions, focusing first on the nuclear one. $C_{\mathrm{n}}$ arises from the ${ }^{167} \mathrm{Er}$ nuclear magnetic moments $\left({ }^{167} \mathrm{Er}\right.$ is the only non-spinless isotope of Er with $23 \%$ natural abundance), since the contribution of the two $\mathrm{Ti}$ isotopes is negligible, as in the case of $\mathrm{Tb}_{2} \mathrm{Ti}_{2} \mathrm{O}_{7} .{ }^{38}$ Contrary to $\mathrm{Tb}_{2} \mathrm{Ti}_{2} \mathrm{O}_{7}$ the quadrupole interaction is not negligible compared to the Zeeman interaction. This is due to the fact that the quadrupole moment $Q_{167}$ of ${ }^{167} \mathrm{Er}$ is larger than that of ${ }^{159} \mathrm{~Tb}(3.565 \mathrm{vs}$ 1.432 barns) and the gyromagnetic ratio $\gamma_{167}$ of ${ }^{167} \mathrm{Er}$ is much smaller, in absolute value, than that of ${ }^{159} \mathrm{~Tb}(-7.7157 \mathrm{vs}$ 64.31 $\mathrm{Mrad} \mathrm{s}^{-1} \mathrm{~T}^{-1}$ ); see Ref. 41. The Zeeman and quadrupolar Hamiltonians are written

$$
\mathcal{H}_{\text {Zee }}=-\hbar \gamma_{167} \mathbf{I} \cdot \mathbf{B}_{\text {hyp }}
$$

and

$$
\mathcal{H}_{\mathrm{Q}}=\hbar \omega_{\mathrm{Q}}\left[3 I_{z}^{2}-I(I+1)\right],
$$

respectively. In these equations, $\mathbf{I}$ is the ${ }^{167} \mathrm{Er}$ spin operator $(I=7 / 2)$ and $\hbar \omega_{\mathrm{Q}}=\frac{e Q_{167} V_{z z}}{4 I(2 I-1)}$, where $V_{z z}$ is the principal component of the electric field gradient tensor acting on the rare-earth nucleus with $z$ being as before the local threefold axis. The symmetry at the rare-earth site imposes the electricfield gradient to be axial. Because the $\mathrm{Er}^{3+}$ ordered magnetic moments are (nearly) perpendicular to $z$ we shall also take
$\mathbf{B}_{\text {hyp }}$ perpendicular to this axis. As usual $\hbar$ and $e$ stand for the Dirac constant and the proton electric charge, respectively.

The nuclear energy levels are determined after diagonalization of the Hamiltonian $\mathcal{H}_{\mathrm{n}}=\mathcal{H}_{\text {Zee }}+\mathcal{H}_{\mathrm{Q}}$ and $C_{\mathrm{n}}$ is readily obtained. $\mathcal{H}_{\mathrm{n}}$ depends on two parameters, $B_{\text {hyp }}$ and $V_{z z}$. While an estimate for $V_{z z}$ is provided in Appendix B, $B_{\text {hyp }}$ will be a fitting parameter.

The other contribution to the low-temperature specific heat arises from magnons. Low-energy magnons have indeed been observed in neutron scattering experiments. ${ }^{20}$ The dispersion relation $\hbar \omega(\mathbf{q})$ for their lowest energy branch is needed to compute $C_{\mathrm{sw}}$. An approximate expression valid at small wave vectors is

$$
\hbar^{2} \omega^{2}(\mathbf{q})=\hbar^{2} \omega^{2}(q)=\Delta_{\mathrm{sw}}^{2}+\hbar^{2} v_{\mathrm{sw}}^{2} q^{2} .
$$

Here $\Delta_{\mathrm{sw}}$ is the gap energy of the magnon spectrum at the zone center and $v_{\mathrm{sw}}$ is the magnon velocity. We note that a dispersion relation has recently been proposed for $\mathrm{Er}_{2} \mathrm{Ti}_{2} \mathrm{O}_{7}$ in the framework of linear spin-wave theory. ${ }^{26}$ The applicability of this theory in frustrated systems might be questionable as recently discussed in the case of the triangular lattice. ${ }^{42}$ Still, the model of Ref. 26 leads to an anisotropic dispersion relation. The resulting specific heat depends on a single magnon velocity which is the geometrical mean of the three magnon velocities along orthogonal axes. In our model it corresponds to $v_{\mathrm{sw}}$.

When $\Delta_{\mathrm{sw}}$ is negligible, the magnon specific heat $C_{\mathrm{sw}}$ can be computed in the temperature range where only small wave vectors are at play, i.e., when Eq. (10) applies. The expected $T^{3}$ law for $C_{\mathrm{sw}}$ is derived:

$$
C_{\mathrm{sw}}=\mathcal{A} T^{3} \quad \text { with } \quad \mathcal{A}=\frac{\pi^{2}}{120} \mathcal{N}_{\mathrm{A}} \frac{k_{\mathrm{B}}^{4} a^{3}}{\hbar^{3} v_{\mathrm{sw}}^{3}},
$$

where $\mathcal{N}_{\mathrm{A}}$ is Avogadro's constant. This result only holds at sufficiently low temperature.

Having established the theoretical background, we now perform the specific-heat data analysis. We shall do it in two steps. We first attempt to determine whether a $T^{3}$ behavior can be observed. A fit to the measured specific heat at the lowest temperatures for which the magnon contribution should be negligible enables us to estimate $C_{\mathrm{n}}(T)$ and then to subtract it from the measured heat capacity. The resulting electronic heat capacity, $C_{\text {elec }}$, is presented in the main panel of Fig. 7 for our data and the ones of Siddharthan et al. It follows nicely a $T^{3}$ law in a restricted temperature range, but deviates above $\approx T_{\mathrm{N}} / 2.5$, in contrast to published results. ${ }^{19,34}$ This observation justifies identifying $C_{\text {elec }}$ with $C_{\mathrm{sw}}$. The $T^{3}$ behavior is not expected to be seen at low temperature if the energy gap is appreciable. The effect of the gap might be seen around $T=0.2 \mathrm{~K}$; see Fig. 7. However, $C_{\text {elec }}$ becomes very small at that temperature and difficult to measure as reflected by the distribution of the $C_{\text {elec }}$ data. Hence, we cannot determine whether a gap is present from this plot. Numerically, since we can identify $\mathcal{B}$ given in the caption of Fig. 7 with $\mathcal{A}$ of Eq. (11), we get $v_{\text {sw }}=$ $86(2) \mathrm{m} \mathrm{s}^{-1}$.

The second step for the interpretation of the specific-heat data consists in fitting the measured specific heat to the sum $C_{\mathrm{n}}+C_{\mathrm{sw}}$. This sum depends on three parameters $\Delta_{\mathrm{sw}}, v_{\mathrm{sw}}$, and $B_{\text {hyp }}$. The fit is shown in the inset of Fig. 7. Its temperature range is restricted on the high-temperature side because of the 
requirement that only the low-energy magnons determine the value of the integral. Two solid lines are drawn in the figure since the two data sets are slightly different at low temperature. Both sets can be fitted to a range of gaps extending from nearly zero to an upper bound. We find $\Delta_{\mathrm{sw}} / k_{\mathrm{B}} \leqslant 0.5$ (1) $\mathrm{K}$ for both data sets. This is consistent with the value of Sosin et al. ${ }^{34} \mathrm{We}$ also derive $v_{\mathrm{sw}}=84$ (2) and 82 (2) $\mathrm{m} \mathrm{s}^{-1}$ and $B_{\text {hyp }}=345$ (10) and 305 (5) $\mathrm{T}$ for the Siddharthan et al. data and our data, respectively. Note that $B_{\text {hyp }}$ depends very little on the actual value chosen for $V_{z z}$.

We now discuss these results, starting with the bound on the spin gap energy. This bound is really small and might be surprising at a first sight given the strong magnetic anisotropy of $\mathrm{Er}_{2} \mathrm{Ti}_{2} \mathrm{O}_{7}$. However, the $\mathrm{Er}^{3+}$ magnetic moment lies at a polar angle of nearly $90^{\circ}$ with respect to the local threefold axis. This angle is imposed by the relatively strong crystal-field interaction. There is still a continuous degree of freedom for the azimuthal angle. The magnetic order breaks this rotational symmetry and $\Delta_{\mathrm{sw}}$ is a measure of the residual anisotropy energy. We note that the upper bound value for $\Delta_{\mathrm{sw}}$ is in the expected range if it arises from the dipole interaction between the $\mathrm{Er}^{3+}$ magnetic moments.

We examine now the magnon velocity value and tentatively relate it with the exchange integral introduced in Eq. (6). For this purpose we resort to the phenomenological dispersion relation

$$
\hbar^{2} \omega^{2}(\mathbf{q})=\hbar^{2} \omega^{2}(q)=\Delta_{\mathrm{sw}}^{2}+\left[\mathcal{I} z_{\mathrm{nn}} J \sin (q d)\right]^{2},
$$

where $d$ is the distance between two magnetic atoms. In fact, had we written $\sin \left(q^{Z} d\right)$ instead of $\sin (q d)$, Eq. (12) would give the dispersion relation of an antiferromagnetic chain running along the $Z$ direction and of lattice parameter $d$ for which the number of nearest neighbors is $z_{\mathrm{nn}}=2$; see for example Ref. 43. Here we shall take $d$ as the distance between two Er atoms; i.e., $d=a /(2 \sqrt{2})$. Identifying the small $q$ expansion of Eq. (12) with Eq. (10) we have

$$
v_{\mathrm{sw}}=\frac{15}{2 \sqrt{2}} a \frac{\mathcal{I}}{\hbar},
$$

using $J=15 / 2$. This relation leads to $\mathcal{I} / k_{\mathrm{B}}=0.118(4) \mathrm{K}$, a value in reasonable agreement with the one derived from the Curie-Weiss constant; see Sec. IV A. We shall return to the interpretation of the magnon velocity at the end of Sec. V B once a nearest-neighbor Hamiltonian consistent with the symmetry of the lattice has been introduced.

It is possible to get interesting information from the $B_{\text {hyp }}$ values. Using the hyperfine constant which is $87(1) \mathrm{T} / \mu_{\mathrm{B}},{ }^{44}$ the magnetic moment at the origin at the field can be obtained. We derive for the moment $4.0(2)$ and $3.5(1) \mu_{\mathrm{B}}$ from the Siddharthan et al. measurement and ours, respectively. The latter value is consistent with the neutron result. ${ }^{31}$ Hence, contrary to $\mathrm{Tb}_{2} \mathrm{Sn}_{2} \mathrm{O}_{7}$ (Ref. 45) there is no reduction of the hyperfine field at the ${ }^{167} \mathrm{Er}$ nuclei due to electronic spin dynamics. This means that the characteristic time for the electronic spin-flip is substantially larger than the ${ }^{167} \mathrm{Er}$ spin-lattice relaxation time. ${ }^{11}$

We now turn our attention to the critical behavior of the specific heat in the paramagnetic phase. In Fig. 8 we display our data using a reduced temperature scale. We expect to observe

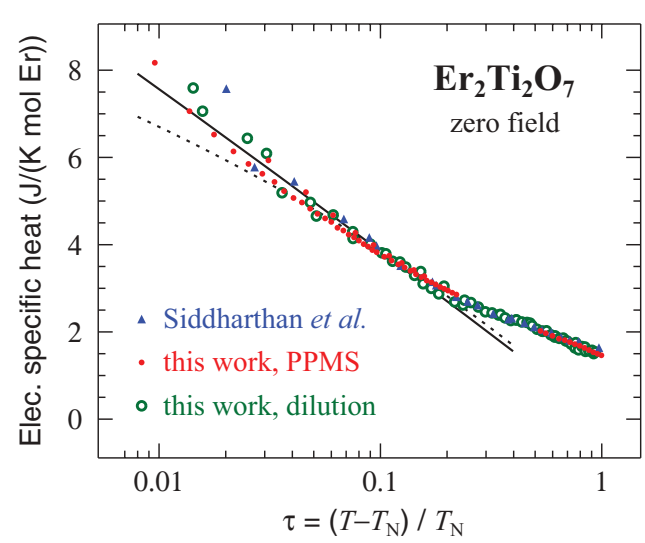

FIG. 8. (Color online) Zero-field specific heat of $\mathrm{Er}_{2} \mathrm{Ti}_{2} \mathrm{O}_{7}$ versus the reduced temperature parameter $\tau$ in the paramagnetic regime for three data sets. The full (dotted) line is the prediction of Eq. (14) for $\alpha=-0.015(-0.134)$. For $\alpha=-0.015$ we find $C_{\mathrm{sh}}=1.7$ (1) $\mathrm{J} \mathrm{K}^{-1}$ $\mathrm{mol}^{-1}$. The critical regime is observed up to $\tau \simeq 0.2$.

the usual power-law critical behavior: $:^{46,47}$

$$
C_{\text {elec }}(T)=\frac{C_{\text {sh }}}{\alpha}\left[\left(\frac{T-T_{\mathrm{N}}}{T_{\mathrm{N}}}\right)^{-\alpha}-1\right],
$$

where $C_{\text {sh }}$ is a constant and $\alpha$ the specific-heat critical exponent. By definition, $C_{\text {elec }}(T)$ has a maximum at $T_{\mathrm{N}}$. This enables us to determine $T_{\mathrm{N}}$, as already mentioned in Sec. III. The exponent $\alpha$ is expected to be $\alpha=-0.015$ and -0.134 for the three-dimensional $X Y$ and Heisenberg magnets, respectively. As seen in Fig. 8, Eq. (14) provides a better account of the data for the $X Y$ case, as expected.

Before leaving this section, we discuss the entropy variation of our system using our specific-heat results. We have extended the specific-heat measurement $C_{\mathrm{p}}$ of $\mathrm{Er}_{2} \mathrm{Ti}_{2} \mathrm{O}_{7}$ up to approximately $20 \mathrm{~K}$ and measured the specific heat of the isostructural nonmagnetic compound $\mathrm{Y}_{2} \mathrm{Ti}_{2} \mathrm{O}_{7}$ in the same temperature range. The sum of the contributions from the nuclear moments and the lattice, the latter being estimated from scaling the $\mathrm{Y}_{2} \mathrm{Ti}_{2} \mathrm{O}_{7}$ result, ${ }^{48}$ is presented in the inset of Fig. 9, as well as $C_{\mathrm{p}}(T)$. The resulting $C_{\mathrm{elec}}(T)$ obtained

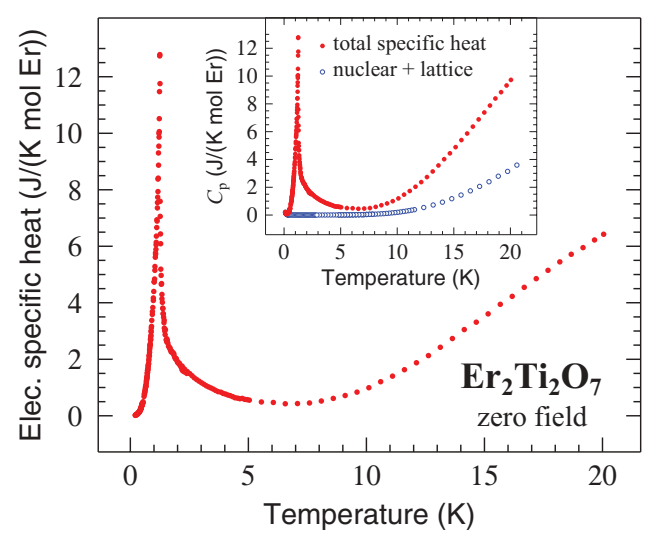

FIG. 9. (Color online) The electronic specific heat of $\mathrm{Er}_{2} \mathrm{Ti}_{2} \mathrm{O}_{7}$ in an extended temperature range. In the inset are displayed the total specific heat $\left(C_{\mathrm{p}}\right)$ of $\mathrm{Er}_{2} \mathrm{Ti}_{2} \mathrm{O}_{7}$ and the sum of the estimated nuclear and lattice contributions. 


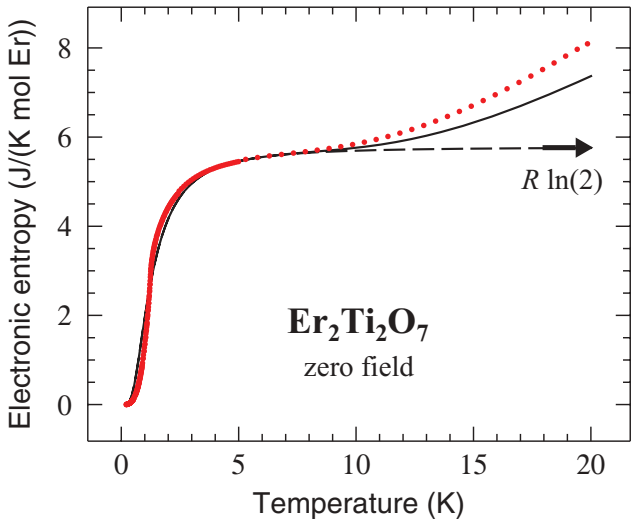

FIG. 10. (Color online) The variation of the electronic entropy $\Delta S_{\text {elec }}(T)$ of $\operatorname{Er}_{2} \mathrm{Ti}_{2} \mathrm{O}_{7}$ versus temperature $T$. The solid line is computed assuming two singlet states at energies corresponding to 0 and $2.85 \mathrm{~K}$ and two doublets at 74 and $85.8 \mathrm{~K}$. A signature of the two doublets has been seen by inelastic neutron scattering (Ref. 19). The ground-state doublet has been split to account for the magnetic order of $\mathrm{Er}_{2} \mathrm{Ti}_{2} \mathrm{O}_{7}$ below $T_{\mathrm{N}}$, with the splitting taken as a fitting parameter. Admittedly, this is a very rough description of the physics. The dashed line is the result of the computation of $\Delta S_{\text {elec }}(T)$ when only the first two levels are taken into account.

from subtracting the contributions of the nuclear moments and the lattice to $C_{\mathrm{p}}(T)$ is displayed in the main panel of Fig. 9. In Fig. 10 we present $\Delta S_{\text {elec }}(T)$, which is the temperature variation of the electronic entropy obtained by integrating $C_{\text {elec }}\left(T^{\prime}\right) / T^{\prime}$ from $0.115 \mathrm{~K}$, the lowest measured temperature, to the temperature of interest $T$. Remarkably, $\Delta S_{\text {elec }}(T)$ reaches the $R \ln (2)$ value at approximately $8 \mathrm{~K}$. This is the entropy variation expected for an isolated doublet state. Therefore the residual entropy left as $T \rightarrow 0$ is extremely small if any. For temperatures above $8 \mathrm{~K}, \Delta S_{\text {elec }}(T)$ keeps increasing owing to the contribution of the excited CEF levels. This is clearly shown in Fig. 10, where a comparison is made between the results of computations of $\Delta S_{\text {elec }}(T)$ either taking into account the first excited CEF levels or neglecting them.

\section{Specific heat under an external magnetic field}

We have constructed the phase diagram of $\mathrm{Er}_{2} \mathrm{Ti}_{2} \mathrm{O}_{7}$ in the field-temperature plane using specific-heat data. Examples of measurements are shown in Fig. 11. For a given external field we have determined the temperature at which the specific heat displays a maximum. The position of the maximum as a function of the field intensity for a given field orientation relative to the crystal axes is displayed in Fig. 12. Our results are consistent with the ones already published, ${ }^{20,34}$ but only qualitatively. Note that here we establish the phase diagram for the three main directions of a cubic compound. These data suggest a quantum critical point to be present slightly above $2 \mathrm{~T}$ and to be dependent on the field orientation.

\section{MICROSCOPIC TECHNIQUE MEASUREMENTS}

Single crystals of $\mathrm{Er}_{2} \mathrm{Ti}_{2} \mathrm{O}_{7}$ have been studied by two microscopic experimental techniques: positive muon spin

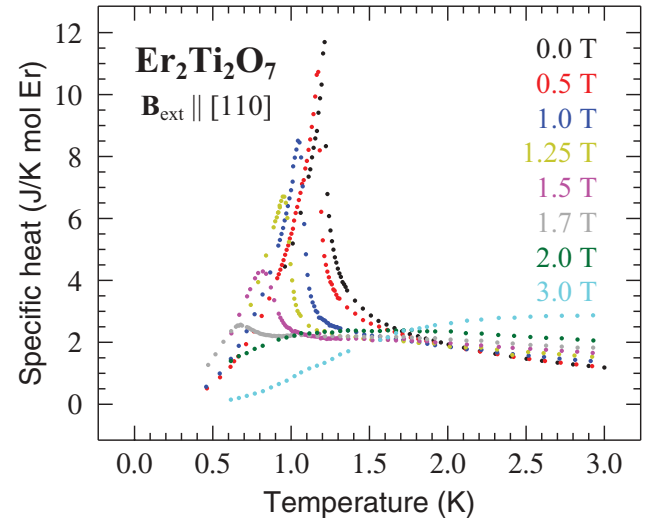

FIG. 11. (Color online) Temperature dependence of the specific heat of $\mathrm{a} \mathrm{Er}_{2} \mathrm{Ti}_{2} \mathrm{O}_{7}$ single crystal for different magnetic field intensities applied along [110]. The maximum of the specific-heat peak moves to lower temperatures as the field increases up to $1.7 \mathrm{~T}$. No peak is observed when the field strength is above $1.7 \mathrm{~T}$.

relaxation ( $\mu \mathrm{SR})$ and neutron scattering. We shall first discuss the $\mu \mathrm{SR}$ results.

\section{A. $\mu \mathrm{SR}$}

In the longitudinal geometry that we have used, a $\mu \mathrm{SR}$ spectrum recorded in the magnetically ordered state of a crystal is expected to display either (i) at least one damped oscillation if the initial muon beam polarization is not parallel to the spontaneous field at the muon site, or (ii) a missing fraction if the oscillation cannot be resolved. ${ }^{49}$ None of these two possibilities was observed at ISIS or $\mathrm{S} \mu \mathrm{S}$. The zero-field spectrum recorded at $21 \mathrm{mK}$ is displayed in Fig. 13; the spectral shape shows little change up to $\approx 0.5 \mathrm{~K}$. The same type of spectra was also observed for different orientations of the initial muon beam polarization relative to the crystal axes. Hence, the absence of oscillation cannot be attributed to the initial muon beam polarization which would be parallel to the internal field. This situation would moreover be unexpected because of the four equivalent symmetry $\langle 111\rangle$ axes at the $\mathrm{Er}^{3+}$ site. In addition to the absence of oscillation, the shape of the zero-field spectra is extremely unusual. Indeed, the spectral slope is quasiconstant up to $\approx 0.4 \mu \mathrm{s}$, then it increases and

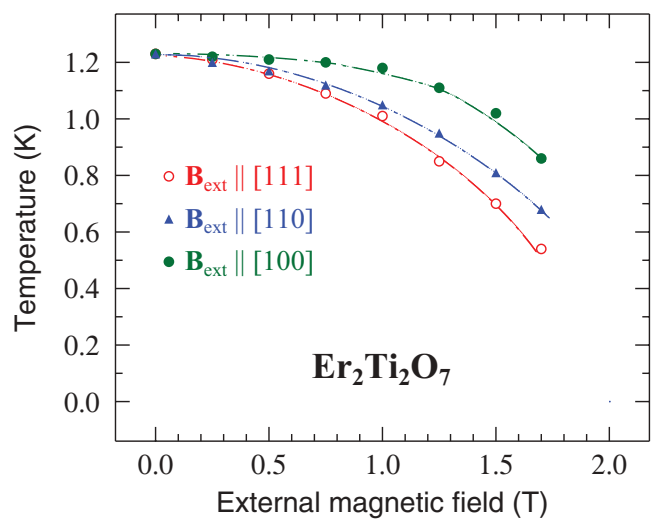

FIG. 12. (Color online) The phase diagram derived from specificheat measurements for the three main crystal directions of cubic $\mathrm{Er}_{2} \mathrm{Ti}_{2} \mathrm{O}_{7}$. The dashed-dotted lines are guides to the eye. 


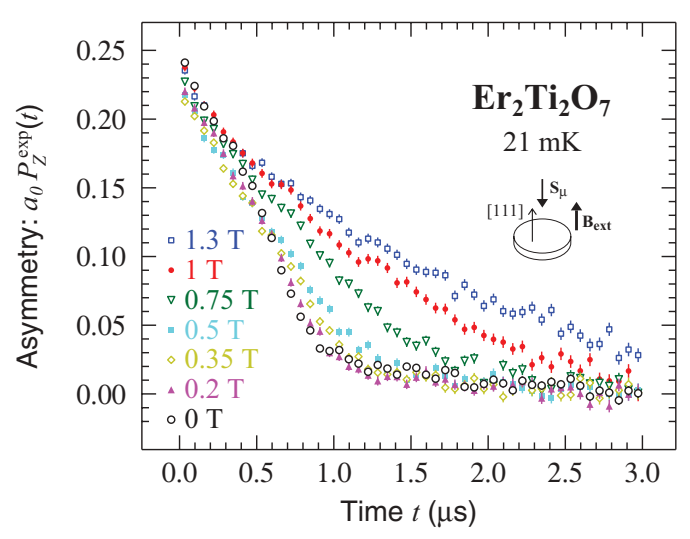

FIG. 13. (Color online) $\mu \mathrm{SR}$ spectra of $\mathrm{a}_{\mathrm{Er}_{2}} \mathrm{Ti}_{2} \mathrm{O}_{7}$ crystal taken at $\mathrm{S} \mu \mathrm{S}$ with the initial muon beam polarization parallel to the [111] crystal direction. The spectra were recorded in zero and various longitudinal fields as indicated in the figure.

eventually monotonically decreases above $\approx 0.7 \mu$ s. This kind of behavior drastically differs from the usual relaxation spectra, which are characterized by a monotonically decreasing slope, as, e.g., in the common exponential relaxation. Neither does it remind us of the shape of spectra associated with a static or quasistatic field distribution at the muon. At this stage we cannot actually conclude whether the spectral shape is mainly influenced by a static field distribution or dynamical effects. We shall come back to this point when commenting on the data recorded in applied fields.

Since no spontaneous muon precession is observed, it is tempting to make an analogy with $\mathrm{Tb}_{2} \mathrm{Sn}_{2} \mathrm{O}_{7}$ for which there is also no detected oscillation in the magnetically ordered state. ${ }^{6}$ This analogy would be completely justified if the zero-field spectrum were exponential. However, we have just remarked that it is obviously not the case. It is not Gaussian either, as first suggested. ${ }^{50}$ A close look at the data in Ref. 50 shows that the zero-field relaxation is in fact consistent with the one we observe.

Before discussing further the significance of our result, it is worthwhile to consider the longitudinal field spectra shown in Fig. 13. First note that with the considered field intensities the compound has a priori not crossed the phase diagram boundary displayed in Fig. 12. While the application of fields below $0.5 \mathrm{~T}$ has little influence on the spectral shape, it is not the case for higher fields. Interestingly, for the highest fields shown in the figure, the spectra tend to be described by an exponential function. ${ }^{51}$ This implies that the muon repolarization is not at the origin of the field dependence of the spectra and that dynamics is mainly influencing the muon response in $\mathrm{Er}_{2} \mathrm{Ti}_{2} \mathrm{O}_{7}$. The time scale of this dynamics can be roughly estimated. For this purpose we have recourse to the Lorentzian field dependence of the exponential relaxation rate $\lambda_{Z}$ in the motional narrowing, i.e., fast fluctuation limit. ${ }^{49,52}$ In this model the relaxation rate in a field $B_{\text {ext }}$ is such that $\lambda_{Z}\left(B_{\text {ext }}\right) / \lambda_{Z}\left(B_{\text {ext }}=0\right)=1 / 2$ for $B_{\text {ext }}=B_{1 / 2}=$ $1 /\left(\gamma_{\mu} \tau_{\mathrm{f}}\right)$. Here $\tau_{\mathrm{f}}$ is the fluctuation time of the spins and $\gamma_{\mu}=$ $851.615 \mathrm{Mrad} \mathrm{s}^{-1} \mathrm{~T}^{-1}$ is the muon gyromagnetic ratio. Taking $1 \mathrm{~T}$ as an order of magnitude for $B_{1 / 2}$ we find $\tau_{\mathrm{f}} \approx 10^{-9} \mathrm{~s}$.

We have therefore established that the muons are probing dynamical fields. No oscillation is detected in zero field because the mean field at the muon site does not keep a constant value for a time sufficiently long for a muon spin precession to be observed. ${ }^{6}$ We denote this field as $\mathbf{B}_{\text {fluc }}$. It arises from the dipole interaction of the muon magnetic moment with the $\mathrm{Er}^{3+}$ magnetic moments. Given the size of the $\mathrm{Er}^{3+}$ magnetic moment, we estimate $B_{\text {fluc }}$ in the range 0.1-0.2 T.

With our knowledge for $\tau_{\mathrm{f}}$ and $B_{\text {fluc }}$, we find $\tau_{\mathrm{f}} \gamma_{\mu} B_{\text {fluc }}=$ $B_{\text {fluc }} / B_{\text {ext }} \ll 1$. For the simple model of $\mathbf{B}_{\text {fluc }}$ flipping from parallel to antiparallel to an axis perpendicular to the initial muon spin polarization $\mathbf{S}_{\mu}$, we would expect the zero-field relaxation to be exponential, ${ }^{49}$ as found for $\mathrm{Tb}_{2} \mathrm{Sn}_{2} \mathrm{O}_{7}$. However, experimentally this is not the case. This is not surprising given the fact that the magnetic diffraction profiles are complex. Their shape is ascribed to the coexistence of long- and short-range dynamical correlations; see our discussion of Fig. 2. To the distribution of correlation lengths must correspond distributions of spin-spin and spin-lattice relaxation rates. This may explain the exotic shape of the zero-field $\mu \mathrm{SR}$ relaxation function.

Relative to $\mathrm{Tb}_{2} \mathrm{Sn}_{2} \mathrm{O}_{7}$, the fluctuations probed by $\mu \mathrm{SR}$ in $\mathrm{Er}_{2} \mathrm{Ti}_{2} \mathrm{O}_{7}$ are slower by roughly an order of magnitude. From neutron spin-echo measurements it is known that in the ordered state of $\mathrm{Tb}_{2} \mathrm{Sn}_{2} \mathrm{O}_{7}$ spin correlations near the zone center are static ${ }^{37}$ within the technique time scale, while they are dynamical in nature far outside the center of the zone. ${ }^{53} \mathrm{It}$ would be worthwhile to examine the dynamics of the magnetic correlations in $\mathrm{Er}_{2} \mathrm{Ti}_{2} \mathrm{O}_{7}$ with the neutron spin-echo technique.

From the analysis of the nuclear specific heat in Sec. IV B, it was inferred that the ratio of $\tau_{\mathrm{f}}$ to the nuclear spin-lattice relaxation time was larger in $\mathrm{Er}_{2} \mathrm{Ti}_{2} \mathrm{O}_{7}$ than in $\mathrm{Tb}_{2} \mathrm{Sn}_{2} \mathrm{O}_{7}$. Assuming the spin-lattice relaxation times in the two compounds to be similar, the nuclear specific-heat data are consistent with the results of the analysis of the $\mu$ SR spectra.

\section{B. Neutron scattering}

Paramagnetic correlations were studied by diffuse neutron scattering in $\mathrm{Er}_{2} \mathrm{Ti}_{2} \mathrm{O}_{7}$ crystals. Two scattering planes were investigated: $(h, k, 0)$ and $(h, k, k)$ at $2.00(3)$ and $1.47(3) \mathrm{K}$, respectively. For the first (second) one a graphite (copper) monochromator was used delivering neutrons of wavelength 2.377 (1.275) A. No energy analysis of the scattered beam was performed. In order to deal with magnetic correlations only, additional maps were recorded at $50 \mathrm{~K}$ for both geometries and they were subtracted from the corresponding low-temperature counterparts. The resulting two maps were divided out by the square modulus of the $\mathrm{Er}^{3+}$ magnetic form factor. ${ }^{54} \mathrm{The}$ effect of the form factor is modest: no more than $20 \%$ on the vast majority of the data. The resulting experimental data are displayed in Fig. 14. It is to be noted that the intensities are negative for large regions in the two planes. This reflects the fact that the wave vector independent scattering associated with the CEF states of the $\mathrm{Tb}^{3+}$ ions is larger at $50 \mathrm{~K}$ than at low temperature.

Before attempting a quantitative analysis of the maps, a qualitative discussion is worthwhile. We first note the almost vanishing $(2,2,2)$ spot. This means that the ferromagnetic correlations are negligible. We observe a hexagonal scattering loop in the plane $(h, k, k)$ around the $(2,2,2)$ position. Such a type of scattering is reminiscent of the intensity measured in the cubic spinel $\mathrm{ZnCr}_{2} \mathrm{O}_{4}$ (Ref. 55), in which the $\mathrm{Cr}$ ions also form a lattice of corner-sharing tetrahedra. However, in 

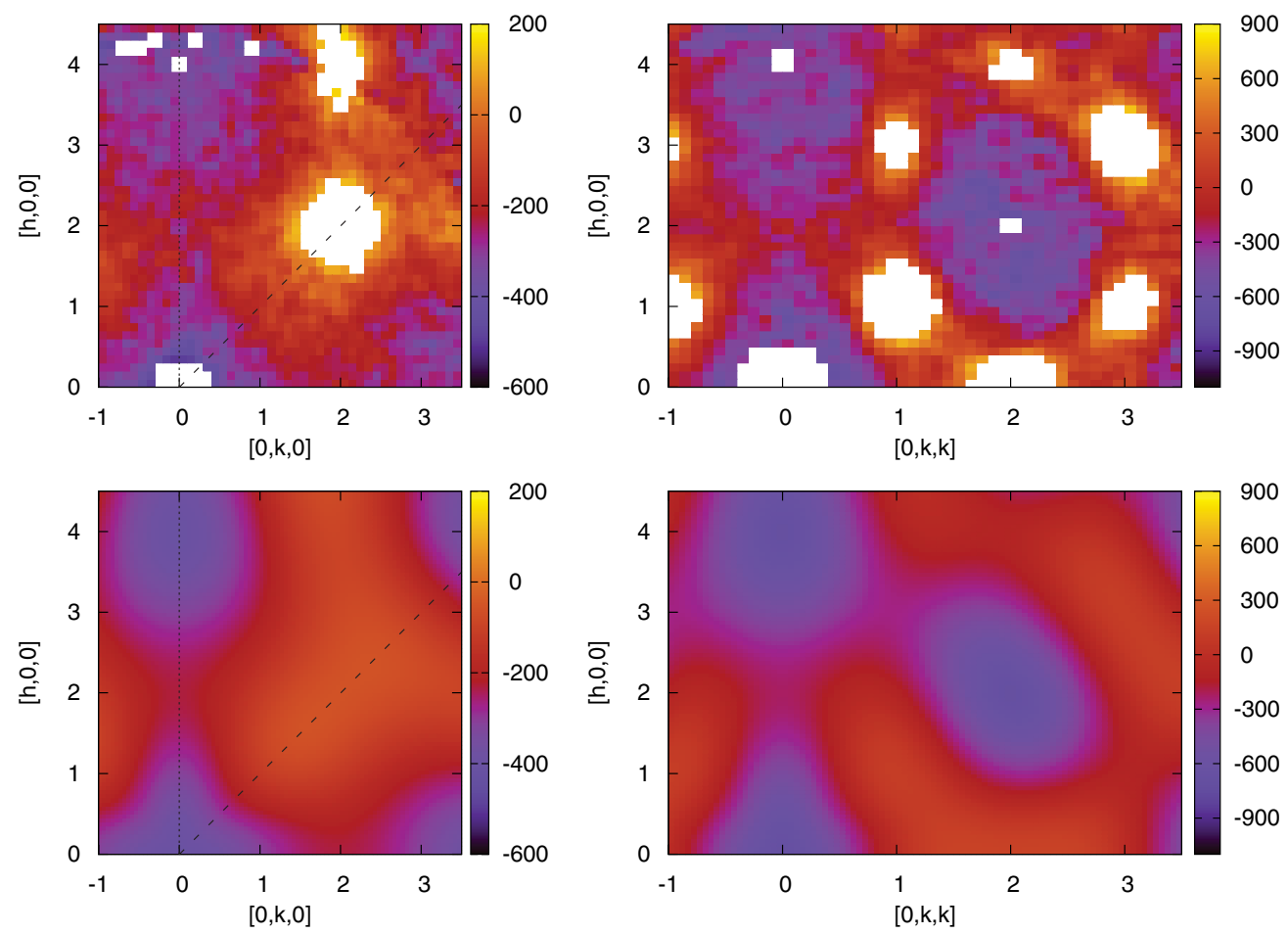

FIG. 14. (Color online) Top two panels: Magnetic diffuse neutron scattering intensity recorded for a crystal of $\mathrm{Er}_{2} \mathrm{Ti}_{2} \mathrm{O}_{7}$ in the reciprocal $(h, k, 0)$ and $(h, k, k)$ planes at $2.00(3)$ and $1.47(3) \mathrm{K}$, respectively. The positions in the reciprocal lattice are in $2 \pi / a$ units, where $a$ is the lattice parameter of the cubic unit cell. These maps are obtained as explained in the main text. To preserve the maps appearance, pixels with off-scale intensities, e.g., pixels influenced by Bragg reflections and critical scattering, as well as pixels located near the origin of the reciprocal lattice have been graphically eliminated: They are represented in white color. Bottom two panels: $(h, k, 0)$ and $(h, k, k)$ magnetic correlation maps computed with the tetrahedron model explained in the main text. The comparison between the theoretical and experimental maps displayed above enables us to derive information on the $\mathrm{Er}_{2} \mathrm{Ti}_{2} \mathrm{O}_{7}$ interaction constants. The lines drawn in the $(h, k, 0)$ maps indicate the position of the cuts shown in Fig. 16.

the latter case the loop is in the plane $(h, k, 0)$ and centered around $(2,2,0)$. The scattering properties are therefore quite different for the two compounds. This reflects the difference in magnetic symmetry. The origin of the loops observed in $\mathrm{ZnCr}_{2} \mathrm{O}_{4}$, which were originally interpreted in terms of weakly interacting hexagonal spin clusters, is now taken as the signature of extended exchange interactions for spin-ice and isotropic systems. ${ }^{56,57}$ In the following we show that the scattering loop in $\mathrm{Er}_{2} \mathrm{Ti}_{2} \mathrm{O}_{7}$ can basically be taken as a fingerprint of the properties of the exchange interactions within a single tetrahedron.

The discussion of the experimental results will be carried out in two steps. We shall first evaluate the magnetic correlation length at the temperature of the measurements and then analyze the maps using a four-spin Hamiltonian.

\section{Magnetic correlation length}

Here we determine the correlation length of the critical magnetic correlations. For this purpose we consider the scattered intensity measured in the vicinity of the reciprocal positions $\mathbf{q}_{(h, k, l)}=\mathbf{q}_{(2,2,0)}$ and $\mathbf{q}_{(1,1,1)}$ at $T=2.00$ and $1.47 \mathrm{~K}$, respectively; see Fig. 15. This critical scattering intensity is described by the sum of a Lorentzian function and a constant:

$$
\mathcal{L}\left(\left|\mathbf{q}-\mathbf{q}_{(h, k, l)}\right|\right)=\frac{I_{\mathrm{L}}}{1+\left|\mathbf{q}-\mathbf{q}_{(h, k, l)}\right|^{2} / \kappa_{\mathrm{m}}^{2}}+I_{0},
$$

where $\kappa_{\mathrm{m}}$ is the inverse of the magnetic correlation length. The parameter $I_{\mathrm{L}}$ accounts for the magnitude of the Lorentzian, while $I_{0}$ refers to a neutron intensity which is not related to critical scattering. Since at the temperature of experiments, the width of the critical magnetic scattering curve is much larger than the instrumental resolution, the convolution of Eq. (15) by the resolution function is unnecessary. The fits shown in Fig. 15 yield the magnetic correlation lengths $\xi_{\mathrm{m}}=\kappa_{\mathrm{m}}^{-1}=$ $3.6(2)$ and $6.6(5) \AA$ for the $(2,2,0)$ and $(1,1,1)$ reflections measured at 2.00 and $1.47 \mathrm{~K}$, respectively. As expected, $\xi_{\mathrm{m}}$ shoots up as the sample is cooled toward the transition. These two values are comparable with the $\mathrm{Er}^{3+}-\mathrm{Er}^{3+}$ ion distance $d=$ $3.56 \AA$ A Hence the analysis of the experimental maps shown in Fig. 14 can be performed considering the spin correlations within a single tetrahedron. This is the basis for our quantitative interpretation which is exposed below.

\section{Analysis of the diffuse scattering maps}

While our analysis of the magnetic scattering intensity in the vicinity of reciprocal lattice positions at low temperature shows that the measured wave vector dependence probes shortrange correlations, a wave vector independent scattering is also observed; see Fig. 15. This scattering reflects local physics, for example of crystal-field nature. Denoting $M(\mathbf{q})$ a measured 


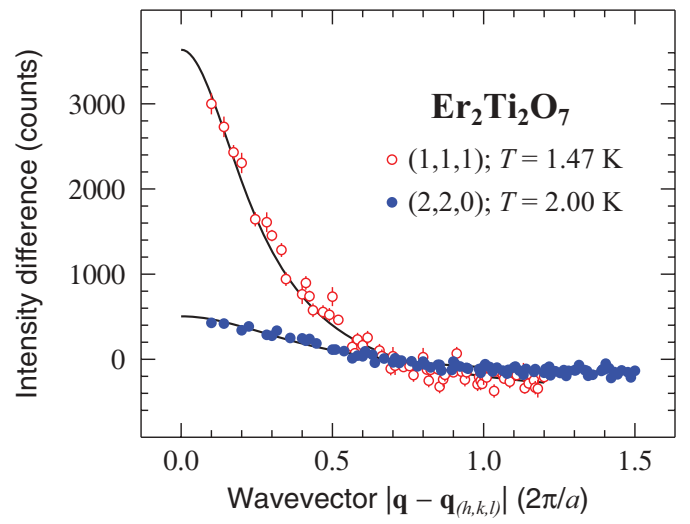

FIG. 15. (Color online) Magnetic scattering intensity versus wave vector in the vicinity of the two reciprocal lattice positions $\mathbf{q}_{(h, k, l)}$ where $(h, k, l)=(1,1,1)$ and $(2,2,0)$, respectively. The wave vector unit is $2 \pi / a$ where $a$ is the cube edge. The data are obtained from the intensity differences shown in Fig. 14 by averaging the data at reciprocal space points located at equal distance from the two lattice positions. Two requirements were considered when choosing these two positions: They do not lie close to the boundary of the maps and the magnetic intensity is relatively important. The lines are results from fits of Eq. (15) to the data.

magnetic scattering map, we write

$$
M(\mathbf{q})=S_{\text {shift }}+S_{\text {scale }} N(\mathbf{q})
$$

where $S_{\text {shift }}$ accounts for the wave vector independent scattering, $S_{\text {scale }}$ gives the scale of the wave vector dependent magnetic intensity, and $N(\mathbf{q})$ is the prediction of our model that we describe now.

In Sec. II we have mentioned that the $\mathrm{Er}^{3+}$ crystal-field ground-state doublet is well isolated since the first excited doublet is at about $74 \mathrm{~K}$ above the ground state in temperature units. Therefore for low-temperature measurements, such as discussed here, it is a reasonable approximation to describe an $\mathrm{Er}^{3+}$ ion as an effective $S=1 / 2$ spin. In Appendix A we discuss the physics of a tetrahedron of effective one-half spins embedded in a pyrochlore lattice. Assuming that only bilinear spin interactions are relevant, the Hamiltonian $\mathcal{H}_{\mathrm{t}}$ describing the interaction between the four effective spins can be written as a linear combination of four invariants; see Eq. (A38). Their weight is gauged by the four parameters $P_{i}, i \in\{1,2,3,4\}$.

The wave vector dependent scattering intensity resulting from a single tetrahedron is proportional to ${ }^{43}$

$$
N(\mathbf{q})=\sum_{m} e^{-E_{m} /\left(k_{\mathrm{B}} T\right)} f_{m}(\mathbf{q}),
$$

where

$$
\begin{aligned}
& f_{m}(\mathbf{q}) \\
& \quad=\sum_{\alpha, \beta} \sum_{i, j} \sum_{n}\left(\delta^{\alpha \beta}-\frac{q^{\alpha} q^{\beta}}{q^{2}}\right)\left\langle m\left|S_{i}^{\alpha}\right| n\right\rangle\left\langle n\left|S_{j}^{\beta}\right| m\right\rangle e^{i \mathbf{q} \cdot\left(\mathbf{r}_{j}-\mathbf{r}_{i}\right)} .
\end{aligned}
$$

The Debye-Waller factor is negligible for the region of the reciprocal space investigated and the temperatures at which the measurements were made. The symbols $\alpha, \beta$ refer to global Cartesian coordinates in the cubic unit cell. The relations between the local and global axes are explicitly given in Appendix A 1. The indices $i$ and $j$ stand for the $\mathrm{Er}$ ions

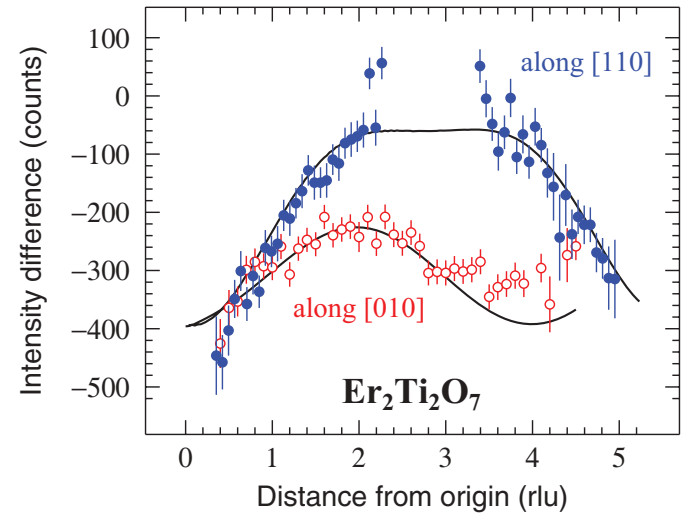

FIG. 16. (Color online) Cuts of the $(h, k, 0)$ maps shown in Fig. 14 along the $[1,1,0]$ and $[0,1,0]$ directions. The circles represent the experimental data and the lines the results of the model. The origin of the horizontal axis is taken at the origin of the reciprocal lattice.

at the corners of the tetrahedron, the positions of which are specified by $\mathbf{r}_{i}$ and $\mathbf{r}_{j}$, and $|n\rangle$ and $|m\rangle$ refer to two of the sixteen single tetrahedron states, whose energy differences lie within the energy range across which the neutron scattering is integrated. The formula in Eq. (18) does not contain the $\mathrm{Er}^{3+}$ form factor since it has already been divided out for the two presented maps.

We have performed a global fit of the two recorded maps. It depends on two $S_{\text {shift }}$ parameters, one per map, a unique scale parameter $S_{\text {scale }}$, and the four Hamiltonian parameters $P_{i}$. The best fit shown in Fig. 14 is achieved with the following interaction constants in kelvin units:

$$
\begin{aligned}
& P_{1} / k_{\mathrm{B}}=10.4(6), \quad P_{2} / k_{\mathrm{B}}=-1.2(5), \\
& P_{3} / k_{\mathrm{B}}=3.0(3), \quad P_{4} / k_{\mathrm{B}}=8.4(1.3) .
\end{aligned}
$$

The quality of the fit can be assessed from cuts of the experimental and model maps as seen in Fig. 16.

The fitting parameters described above were determined from least-squares minimization, i.e., minimizing $\chi^{2}=$ $\left[1 /\left(N_{d}-7\right)\right] \sum_{j}\left(M_{j}-I_{j}\right)^{2} / \sigma_{j}^{2}$ with respect to the fitting parameters. Here $\sigma_{j}$ is the statistical uncertainty on the neutron intensity $I_{j}$ at the $j$ th data point in the set of $N_{d}$ data points and $M_{j}$ is the model prediction (a function of the fitting parameters). An uncertainty $\delta P_{i}$ on $P_{i}$ is obtained by varying the neutron intensity by $\partial I_{j}$ at each of a large sample of the data points (one in eight) and minimizing $\chi^{2}$ in order to find the variation of each fitted parameter $\partial P_{i}$. Then $\delta P_{i}$ is estimated using the relation $\delta P_{i}=\left(1 / N_{d}\right) \sqrt{\sum_{j}\left(\sigma_{j} \partial P_{i} / \partial I_{j}\right)^{2}}$.

We have so far described the estimate of the statistical uncertainties. Three other origins for systematical uncertainties must be considered. First our model describes diffuse scattering and therefore pixels which are sizably influenced by critical scattering should be eliminated in the fitting procedure. For this purpose we have tested the influence of different radius cutoffs around the Bragg points on the $P_{i}$ values. This cutoff effect introduces the largest parameter uncertainties. Then the temperature at which a map is recorded is known with a finite uncertainty. Finally the uncertainty arising from the error bars on the matrix elements $j_{\mathrm{CEF}}$ and $t_{\mathrm{CEF}}$ [see Eq. (2)] has also 
been assessed. The error bars given in Eq. (19) account for these statistical and systematical uncertainties.

It is tempting to extract information from the Curie-Weiss constant using the high-temperature susceptibility formula derived by Ross et al. ${ }^{58}$ However, this should not be done since that formula depends on spectroscopic factors which provide a description of the physics only at low temperature. A high-temperature expansion for the susceptibility is required to further analyze the data of Fig. 5.

At this juncture it is of interest to mention a recent work by Savary et al. on $\mathrm{Er}_{2} \mathrm{Ti}_{2} \mathrm{O}_{7} \cdot{ }^{26}$ These authors analyze the spin wave dispersions measured for different orientations of the wave vector. The experiments were performed for temperature $(30 \mathrm{mK})$ and field $(3 \mathrm{~T})$ values where $\mathrm{Er}_{2} \mathrm{Ti}_{2} \mathrm{O}_{7}$ is a polarized paramagnet. ${ }^{20}$ The analysis of the data involves a nearest-neighbor Hamiltonian related to ours, with a different definition of the Hamiltonian parameters. Using the relations between the two sets of parameters given in Eq. (A41) and assuming $P_{i}=2 \tilde{P}_{i}$, we find for their parameters in kelvin units

$$
\begin{aligned}
& P_{1} / k_{\mathrm{B}}=1.74(1.26), \quad P_{2} / k_{\mathrm{B}}=-0.44(74), \\
& P_{3} / k_{\mathrm{B}}=2.92(34), \quad P_{4} / k_{\mathrm{B}}=9.0(1.0) .
\end{aligned}
$$

These $P_{2}, P_{3}$, and $P_{4}$ values are in very good agreement with ours. However an important difference is observed in $P_{1}$, i.e., the parameter which controls the interaction along the local hard magnetic axis. We have simulated the diffuse scattering intensity obtained from this latter set of parameters and compared it with our experimental data. We obtain an acceptable fit of our data since the confidence parameter is $\chi^{2}=1.44$ to be compared with 1.20 with the parameter set in Eq. (19). At this point it must be noted that our data were recorded in zero external field, contrary to those of Ref. 26, and that the anisotropy ratio $g_{\perp} / g_{\|}=4.3$ that we have adopted is quite larger than the one chosen by Savary et al.: $g_{\perp} / g_{\|}=2.4$.

As already mentioned the dispersion relation of the magnon modes has been computed in the framework of linear spinwave theory. ${ }^{26}$ Neglecting the gap, an assumption which is justified (see Sec. IV B), we can compute the geometric mean $\bar{v}_{\text {sw }}$ of the magnon velocities along the three directions of the Cartesian frame. The expression is

$$
\bar{v}_{\mathrm{sw}}=\frac{\left(P_{4}-P_{1}\right)^{1 / 2}\left(P_{4}-P_{3}\right)^{1 / 6}\left(2 P_{4}+P_{3}\right)^{1 / 3}}{2^{10 / 3} 3^{1 / 2}} \frac{a}{\hbar} .
$$

With the parameters given in Eq. (20) we find $\bar{v}_{\mathrm{sw}}=$ $76(16) \mathrm{m} \mathrm{s}^{-1}$, in good agreement with the values $v_{\mathrm{sw}}=84$ (2) and $82(2) \mathrm{m} \mathrm{s}^{-1}$ deduced in Sec. IV B from the analysis of the specific-heat data.

Concerning the parameters derived from our diffuse scattering maps [Eq. (19)], the magnon velocity cannot be computed since $\left(P_{4}-P_{1}\right)<0$. At first sight, it might suggest that the parameters we infer from the two neutron maps are not reliable. However, the expression written in Eq. (21) is deduced from a linear spin-wave approximation. This approximation might not be reliable for a noncollinear magnet such as $\mathrm{Er}_{2} \mathrm{Ti}_{2} \mathrm{O}_{7}$. This argument is based on theoretical results for triangular magnets. $42,59,60$

\section{SUMMARY OF OUR RESULTS AND DISCUSSION}

In this paper we have argued that the combined analysis of the values of the low-temperature $\mathrm{Er}^{3+}$ magnetic moment and the two spectroscopic factors indicates that the moment can only be perpendicular (or close to perpendicular) to the local [111] axis. This analysis supports the magnetic structure proposed by Champion et al. ${ }^{19}$

The $\mathrm{Er}_{2} \mathrm{Ti}_{2} \mathrm{O}_{7}$ magnetic susceptibility and specific heat have been carefully measured. No magnetic hysteresis has been detected. The excitation gap is extremely small. The critical exponent of the specific heat in the paramagnetic phase is typical for a three-dimensional $X Y$ system. The specific-heat data provide an estimate for the magnon velocity which is in accord with a recently proposed model based on linear spin-wave theory. Finally, concerning the bulk measurements, the magnetic phase diagram in the field-temperature plane has been determined up to $1.7 \mathrm{~T}$ for the three main crystal directions of a cube.

The $\mu$ SR data are consistent with the presence of a spin dynamics in the nanosecond time range in the ordered state. This might be associated with the short-range correlations detected by neutron diffraction in addition to the long-range order. $^{20}$

Diffuse neutron scattering data recorded in the paramagnetic state were analyzed in terms of a Hamiltonian accounting for all bilinear interactions between the spins in a tetrahedron. The four symmetry-allowed interaction constants were determined and three of them were found to be positive, with $P_{1}$ and $P_{4}$ being the largest. Note that the data analysis has assumed the interactions to be limited to the nearest-neighbor $\mathrm{Er}^{3+}$ ions. However, it must be recalled that interactions between further neighbors might be important. For instance they are determinant for the type of magnetic order adopted by $\mathrm{Gd}_{2} \mathrm{Ti}_{2} \mathrm{O}_{7}$ relative to $\mathrm{Gd}_{2} \mathrm{Sn}_{2} \mathrm{O}_{7}$ for which only nearest neighbors seem to matter. ${ }^{9}$ The analysis of neutron scattering data for $\mathrm{Dy}_{2} \mathrm{Ti}_{2} \mathrm{O}_{7}$ also confirms the importance of exchange interactions beyond nearest neighbors. ${ }^{56}$

With the interaction constants and the spectroscopic factors known, it will be interesting to gauge any proposed theoretical phase diagram to the fact that $\mathrm{Er}_{2} \mathrm{Ti}_{2} \mathrm{O}_{7}$ does order magnetically at $T_{\mathrm{N}}=1.23(1) \mathrm{K}$. In addition, any reliable theory must be able to explain the short-range correlations observed below $T_{\mathrm{N}}$ and their nanosecond time scale dynamics. As has been learned from the study of $\mathrm{Tb}_{2} \mathrm{Sn}_{2} \mathrm{O}_{7}$, neutron spin-echo measurements might be useful to further characterize these exotic spin dynamics. ${ }^{37,53}$ The spin dynamics is the key feature which seems to distinguish a frustrated compound such as $\mathrm{Er}_{2} \mathrm{Ti}_{2} \mathrm{O}_{7}$ from a conventional magnet.

\section{ACKNOWLEDGMENTS}

This research project has been partially supported by the European Science Foundation through the Highly Frustrated Magnetism program. The $\mu \mathrm{SR}$ measurements were performed at $\mathrm{S} \mu \mathrm{S}$, Paul Scherrer Institute, Villigen, Switzerland, and at the ISIS facility, Rutherford Appleton Laboratory, Chilton, UK. The neutron data were recorded at the Institut Laue Langevin, Grenoble, France. We thank S. S. Sosin for his contribution to the specific-heat measurements, J. A. Hodges 
for ongoing discussions on Kramers doublets systems, M. E. Zhitomirsky for numerous discussions on frustrated magnets, J. Jerrett for technical assistance, and B. Fåk for a careful reading of the manuscript. S.C. was supported by NSERC of Canada.

\section{APPENDIX A: PHYSICS OF A TETRAHEDRON OF EFFECTIVE ONE-HALF SPINS EMBEDDED IN A PYROCHLORE LATTICE}

Here we present a comprehensive quantum mechanical study of effective one-half spins embedded in a pyrochlore lattice. After specifying the geometry, we describe the invariants which will enable us to build the Hamiltonian of the system. Then we explain the approximation that we use to compute the neutron diffuse scattering patterns. Finally, we compare our to others' equivalent Hamiltonian operators.

\section{Geometry}

For completeness, we first provide a description of a tetrahedron in a pyrochlore lattice and of the spins at its corners. We choose a type A tetrahedron as the primitive unit cell; see Fig. 1. Each corner of the tetrahedron is occupied by a magnetic ion whose relative positions are given by $\mathbf{r}_{1}=$ $\frac{a}{4}(0,0,0), \mathbf{r}_{2}=\frac{a}{4}(1,1,0), \mathbf{r}_{3}=\frac{a}{4}(1,0,1)$, and $\mathbf{r}_{4}=\frac{a}{4}(0,1,1)$, where $a$ is the edge length of the cubic unit cell. The distance between two magnetic ions is $d=a /(2 \sqrt{2})$. At each corner of the tetrahedron there is a local $D_{3 d}$ symmetry, where the $C_{3}$ axis is one of the cube diagonals.

We denote the local $C_{3}$ axis at position $i$ as $z_{i}$ and define $x_{i}$ and $y_{i}$ axes to form a local orthogonal basis. There is obviously some freedom in the choice of the $x_{i}$ and $y_{i}$ axes. Below we list the unit vectors we have chosen for the bases at the four positions. For the first two positions we have

$$
\begin{aligned}
& \hat{\mathbf{x}}_{1}=(1,1,-2) / \sqrt{6}, \quad \hat{\mathbf{x}}_{2}=(-1,-1,-2) / \sqrt{6}, \\
& \hat{\mathbf{y}}_{1}=(-1,1,0) / \sqrt{2}, \quad \hat{\mathbf{y}}_{2}=(1,-1,0) / \sqrt{2}, \\
& \hat{\mathbf{z}}_{1}=(1,1,1) / \sqrt{3}, \quad \hat{\mathbf{z}}_{2}=(-1,-1,1) / \sqrt{3},
\end{aligned}
$$

and at the remaining two positions we have

$$
\begin{aligned}
& \hat{\mathbf{x}}_{3}=(-1,1,2) / \sqrt{6}, \quad \hat{\mathbf{x}}_{4}=(1,-1,2) / \sqrt{6}, \\
& \hat{\mathbf{y}}_{3}=(1,1,0) / \sqrt{2}, \quad \hat{\mathbf{y}}_{4}=(-1,-1,0) / \sqrt{2}, \\
& \hat{\mathbf{z}}_{3}=(-1,1,-1) / \sqrt{3}, \quad \hat{\mathbf{z}}_{4}=(1,-1,-1) / \sqrt{3} .
\end{aligned}
$$

A given spin $\mathbf{S}_{i}$ can be written using the cubic global axes as a basis or the local axes at position $i$. We use uppercase superscripts to indicate components of the global basis,

$$
\mathbf{S}_{i}=S_{i}^{X} \hat{\mathbf{X}}+S_{i}^{Y} \hat{\mathbf{Y}}+S_{i}^{Z} \hat{\mathbf{Z}},
$$

and lowercase subscripts for components of a local frame,

$$
\mathbf{S}_{i}=S_{i x} \hat{\mathbf{x}}_{i}+S_{i y} \hat{\mathbf{y}}_{i}+S_{i z} \hat{\mathbf{z}}_{i} .
$$

Using the definitions above, we find

$$
\begin{gathered}
S_{1 x}=\left(S_{1}^{X}+S_{1}^{Y}-2 S_{1}^{Z}\right) / \sqrt{6}, \\
S_{1 y}=\left(-S_{1}^{X}+S_{1}^{Y}\right) / \sqrt{2}, \\
S_{1 z}=\left(S_{1}^{X}+S_{1}^{Y}+S_{1}^{Z}\right) / \sqrt{3},
\end{gathered}
$$

$$
\begin{gathered}
S_{2 x}=-\left(S_{2}^{X}+S_{2}^{Y}+2 S_{2}^{Z}\right) / \sqrt{6}, \\
S_{2 y}=\left(S_{2}^{X}-S_{2}^{Y}\right) / \sqrt{2}, \\
S_{2 z}=\left(-S_{2}^{X}-S_{2}^{Y}+S_{2}^{Z}\right) / \sqrt{3}, \\
S_{3 x}=\left(-S_{3}^{X}+S_{3}^{Y}+2 S_{3}^{Z}\right) / \sqrt{6}, \\
S_{3 y}=\left(S_{3}^{X}+S_{3}^{Y}\right) / \sqrt{2}, \\
S_{3 z}=\left(-S_{3}^{X}+S_{3}^{Y}-S_{3}^{Z}\right) / \sqrt{3}, \\
S_{4 x}=\left(S_{4}^{X}-S_{4}^{Y}+2 S_{2}^{Z}\right) / \sqrt{6}, \\
S_{4 y}=\left(-S_{4}^{X}-S_{4}^{Y}\right) / \sqrt{2}, \\
S_{4 z}=\left(S_{4}^{X}-S_{4}^{Y}-S_{4}^{Z}\right) / \sqrt{3},
\end{gathered}
$$

and the inverse relations

$$
\begin{gathered}
S_{1}^{X}=S_{1 x} / \sqrt{6}-S_{1 y} / \sqrt{2}+S_{1 z} / \sqrt{3}, \\
S_{1}^{Y}=S_{1 x} / \sqrt{6}+S_{1 y} / \sqrt{2}+S_{1 z} / \sqrt{3}, \\
S_{1}^{Z}=-2 S_{1 x} / \sqrt{6}+S_{1 z} / \sqrt{3}, \\
S_{2}^{X}=-S_{2 x} / \sqrt{6}+S_{2 y} / \sqrt{2}-S_{2 z} / \sqrt{3}, \\
S_{2}^{Y}=-S_{2 x} / \sqrt{6}-S_{2 y} / \sqrt{2}-S_{2 z} / \sqrt{3}, \\
S_{2}^{Z}=-2 S_{2 x} / \sqrt{6}+S_{2 z} / \sqrt{3}, \\
S_{3}^{X}=-S_{3 x} / \sqrt{6}+S_{3 y} / \sqrt{2}-S_{3 z} / \sqrt{3}, \\
S_{3}^{Y}=S_{3 x} / \sqrt{6}+S_{3 y} / \sqrt{2}+S_{3 z} / \sqrt{3}, \\
S_{3}^{Z}=2 S_{3 x} / \sqrt{6}-S_{3 z} / \sqrt{3}, \\
S_{4}^{X}=S_{4 x} / \sqrt{6}-S_{4 y} / \sqrt{2}+S_{4 z} / \sqrt{3}, \\
S_{4}^{Y}=-S_{4 x} / \sqrt{6}-S_{4 y} / \sqrt{2}-S_{4 z} / \sqrt{3}, \\
\\
S_{4}^{Z}=2 S_{4 x} / \sqrt{6}-S_{4 z} / \sqrt{3} .
\end{gathered}
$$

\section{Determination of the invariants}

The interaction Hamiltonian $\mathcal{H}$ between the spins in the lattice is described by bilinear operators of the form $S_{i \alpha} S_{j \beta}$ where $i$ and $j$ are nearest neighbors. The space group $F d \overline{3} m$ allows four different terms for a Kramers ion: ${ }^{61-63}$

$$
\mathcal{H}=\tilde{P}_{1} \mathcal{X}_{1}+\tilde{P}_{2} \mathcal{X}_{2}+\tilde{P}_{3} \mathcal{X}_{3}+\tilde{P}_{4} \mathcal{X}_{4},
$$

where $\tilde{P}_{i}$ are constants and

$$
\begin{gathered}
\mathcal{X}_{1}=-\frac{1}{3} \sum_{\langle i, j\rangle} S_{i z} S_{j z} \\
\mathcal{X}_{2}=-\frac{\sqrt{2}}{3} \sum_{\langle i, j\rangle}\left[\Lambda_{i j}\left(S_{i z} S_{j+}+S_{j z} S_{i+}\right)\right. \\
\left.+\Lambda_{i j}^{*}\left(S_{i z} S_{j-}+S_{j z} S_{i-}\right)\right]
\end{gathered}
$$




$$
\begin{gathered}
\mathcal{X}_{3}=\frac{1}{3} \sum_{\langle i, j\rangle}\left(\Lambda_{i j}^{*} S_{i+} S_{j+}+\Lambda_{i j} S_{i-} S_{j-}\right), \\
\mathcal{X}_{4}=-\frac{1}{6} \sum_{\langle i, j\rangle}\left(S_{i+} S_{j-}+S_{j+} S_{i-}\right) .
\end{gathered}
$$

The sum is over all the pairs of nearest neighbors [see Eq. (6) for an example of the use of the notation in the case of the Heisenberg interaction]. Below we give the expressions of the phase factors $\Lambda_{i j}$ introduced for $\mathcal{X}_{2}$ and $\mathcal{X}_{3}$ :

$$
\begin{gathered}
\Lambda_{12}=\Lambda_{34}=1, \\
\Lambda_{13}=\Lambda_{24}=\varepsilon=\exp \left(\frac{2 \pi i}{3}\right), \\
\Lambda_{14}=\Lambda_{23}=\varepsilon^{*}=\exp \left(\frac{4 \pi i}{3}\right) .
\end{gathered}
$$

Note that the sum of all four invariants is the isotropic exchange interaction $\sum_{i=1}^{4} \mathcal{X}_{i}=\sum_{\langle i, j\rangle} \mathbf{S}_{i} \cdot \mathbf{S}_{j}$ (obtained when $\tilde{P}_{1}=\tilde{P}_{2}=\tilde{P}_{3}=\tilde{P}_{4}$ ).

To get some insight in the $\mathcal{H}$ expression, as an example, we consider the product $\mathbf{S}_{1} \cdot \mathbf{S}_{2}$. Let us first focus on the contribution of the first invariant to this product:

$$
\mathcal{X}_{1}:\left(\frac{-1}{3}\right) \frac{1}{2}\left(S_{1 z} S_{2 z}+S_{2 z} S_{1 z}\right)=-\frac{1}{3} S_{1 z} S_{2 z} .
$$

The contribution of the second invariant is easily found:

$$
\mathcal{X}_{2}:-2 \frac{\sqrt{2}}{3}\left(S_{1 z} S_{2 x}+S_{2 z} S_{1 x}\right)
$$

In the same way the contributions of $\mathcal{X}_{3}$ and $\mathcal{X}_{4}$ can be derived. They are written in terms of the local Cartesian axes. Transforming to the global axes we derive the expected relation

$$
\mathbf{S}_{1} \cdot \mathbf{S}_{2}=S_{1}^{X} S_{2}^{X}+S_{1}^{Y} S_{2}^{Y}+S_{1}^{Z} S_{2}^{Z} .
$$

In its most general form [Eq. (A29)] $\mathcal{H}$ includes symmetric (Heisenberg) and the antisymmetric (Dzyaloshinskii-Moriya) interactions. For completeness, we give the expression of the Dzyaloshinskii-Moriya Hamiltonian. In terms of the invariants, we derive

$$
\mathcal{H}_{\mathrm{DM}}=-E_{\mathrm{t}, \mathrm{DM}}\left(4 \mathcal{X}_{1}-\frac{1}{2} \mathcal{X}_{2}+\mathcal{X}_{3}-2 \mathcal{X}_{4}\right),
$$

where $E_{\mathrm{t}, \mathrm{DM}}$ scales the Dzyaloshinskii-Moriya interaction.

\section{Single-tetrahedron approximation}

In order to compute diffuse neutron scattering patterns exact eigenstates of $\mathcal{H}$ must be used; however, the Hamiltonian given by Eq. (A29) is unsolvable in general. Therefore instead of using the full Hamiltonian (Eq. (A29)), we find exact solutions to a Hamiltonian $\mathcal{H}_{\mathrm{t}}$ restricted to tetrahedra of a single type. This approach, which has been used to analyze diffuse neutron scattering patterns, ${ }^{61,64}$ is a considerable simplification of the original model. It amounts to replacing the sums in Eq. (A29) over all tetrahedra, as is implicit from the definition of the $\chi_{i}$ 's, to a sum over all A-type (or all B-type) tetrahedra (Fig. 1), as explained in Ref. 62. Thus only three of the six nearest neighbors of each Er atom are included in the calculation. Instead of the original interaction constants $\tilde{P}_{i}$ we introduce a similar set of constants $P_{i}$, such that

$$
\mathcal{H}_{\mathrm{t}}=\sum_{\text {A tetrahedra }} P_{1} \mathcal{X}_{1}+P_{2} \mathcal{X}_{2}+P_{3} \mathcal{X}_{3}+P_{4} \mathcal{X}_{4}
$$

Here the sums appearing in the $\chi_{i}$ operators are limited to nearest-neighbor spins belonging to single A tetrahedra, unlike in Eqs. (A30)-(A33). While the full ramifications of this approximation are not understood, at the very least we can estimate that the interaction constants $P_{i}$ are approximately a factor of two larger than $\tilde{P}_{i}$ to compensate for the missing exchange paths in Eq. (A38).

\section{Relations between Hamiltonian parameters}

Other groups have proposed a Hamiltonian for the description of an effective one-half spin system such as $\mathrm{Er}_{2} \mathrm{Ti}_{2} \mathrm{O}_{7}$ for which the rare-earth crystal-field ground state is a Kramer's doublet.

Ross et al. ${ }^{58}$ and Savary et $a l .{ }^{26,65}$ write the Hamiltonian as

$$
\begin{aligned}
\mathcal{H}= & \sum_{\langle i, j\rangle}\left\{J_{z z} S_{i z} S_{j z}-J_{ \pm}\left(S_{i+} S_{j-}+S_{i-} S_{j+}\right)\right. \\
& +J_{ \pm \pm}\left(\gamma_{i j} S_{i+} S_{j+}+\gamma_{i j}^{*} S_{i-} S_{j-}\right) \\
& \left.+J_{z \pm}\left[S_{i z}\left(\zeta_{i j} S_{j+}+\zeta_{i j}^{*} S_{j-}\right)+i \leftrightarrow j\right]\right\},
\end{aligned}
$$

where $\gamma$ and $\zeta$ are $4 \times 4$ matrices:

$$
\zeta=\left(\begin{array}{cccc}
0 & -1 & e^{i \pi / 3} & e^{-i \pi / 3} \\
-1 & 0 & e^{-i \pi / 3} & e^{i \pi / 3} \\
e^{i \pi / 3} & e^{-i \pi / 3} & 0 & -1 \\
e^{-i \pi / 3} & e^{i \pi / 3} & -1 & 0
\end{array}\right), \quad \gamma=-\zeta^{*} .
$$

Taking into account (i) the different labeling in the four sites of the tetrahedron as well as (ii) the different choice for the definition of the axes perpendicular to the local threefold axis which are adopted in these references compared to ours, the relations between the Hamiltonian parameters are

$$
\begin{aligned}
& J_{z z}=-\frac{1}{3} \tilde{P}_{1}, \quad J_{z \pm}=\frac{\sqrt{2}}{3} \tilde{P}_{2}, \\
& J_{ \pm \pm}=\frac{1}{3} \tilde{P}_{3}, \quad J_{ \pm}=\frac{1}{6} \tilde{P}_{4} .
\end{aligned}
$$

As explained in Sec. A 3, we expect $P_{i} \approx 2 \tilde{P}_{i}$.

Considering $\mathrm{Yb}_{2} \mathrm{Ti}_{2} \mathrm{O}_{7}$ and others crystal-field ground-state doublet pyrochlore compounds, Onoda and Tanaka ${ }^{66-68}$ have also used a Hamiltonian equivalent to Eq. (A39), but with slight differences in the Hamiltonian parameters labeling.

Two other research groups have studied the interaction of spins on the pyrochlore lattice with the purpose of extracting values of the interaction constants. However, since their interest was on the analysis of relatively high temperature data, the full angular-momentum $\mathbf{J}_{i}$ was used. ${ }^{69,70}$ Here we have recorded the diffuse scattering intensity at a sufficiently small temperature that it is justified to work within the effective one-half spin framework. It is possible to project the full angular momentum into the ground-state Kramer's doublet. However, it seems that only semiformal relations between the parameters of the effective one-half spin and the full 
angular-momentum models can be derived. ${ }^{58}$ Therefore we do not consider these relations in our analysis in Sec. V B2.

\section{APPENDIX B: ESTIMATE OF THE ELECTRIC FIELD GRADIENT ACTING ON THE ${ }^{167} \mathrm{Er}$ NUCLEUS}

Here we provide an estimate for the principal value of electric field gradient tensor $V_{z z}$. It is required for the computation of the nuclear contribution to the specific heat.

In an insulator $V_{z z}$ is written as the sum of two terms $V_{z z}^{\text {latt }}$ and $V_{z z}^{4 f}$, respectively, modeling the lattice and $4 f$-shell contributions. The former contribution is expressed as $V_{z z}^{\text {latt }}=$ $-\frac{4 A_{2}^{0}}{e} \frac{1-\gamma_{\infty}}{1-\sigma_{2}}$ where $A_{2}^{0}$ is a crystal electric field parameter and $\gamma_{\infty}$ and $\sigma_{2}$ are a Sternheimer coefficient and the screening coeffi- cient of the crystal field, respectively. From the literature values $A_{2}^{0}=41.5(1.1) \mathrm{meV}_{0}^{-2}$ (Ref. 28) where $a_{0}=52.92 \mathrm{pm}$ is the Bohr radius and $\left(1-\gamma_{\infty}\right) /\left(1-\sigma_{2}\right)=210$ (30) (Ref. 71), we obtain $V_{z z}^{\text {latt }}=-1.24(21) \times 10^{22} \mathrm{~V} \mathrm{~m}^{-2}$. The $4 f$-shell contribution is written $V_{z z}^{4 f}=-\frac{e}{4 \pi \varepsilon_{0}} \theta_{2}\left(1-R_{Q}\right)\left\langle r^{-3}\right\rangle_{4 f} E_{4 f} . R_{Q}$ is a Sternheimer coefficient, $\theta_{2}$ is a Stevens coefficient, and $\left\langle r^{-3}\right\rangle_{4 f}$ and $E_{4 f}$ are the expectation values respectively of the cube of the inverse distance between the nucleus and the $\mathrm{Er}^{3+} 4 f$ shell, and the quadrupole operator $3 J_{z}^{2}-J(J+1)$ acting on the $4 f$ shell. As usual, $\varepsilon_{0}$ is the permittivity of free space. From the literature values $R_{Q}=0.29$ (1) (Ref. 71), $\theta_{2}=2.54 \times 10^{-3}$, $\left\langle r^{-3}\right\rangle_{4 f}=11.36 a_{0}^{-3}$ (Ref. 72), and $E_{4 f}=-7.86$ (Ref. 28), we get $V_{z z}^{4 f}=1.6 \times 10^{21} \mathrm{~V} \mathrm{~m}^{-2}$. Summing up the two contributions we obtain $V_{z z}=-1.1(3) \times 10^{22} \mathrm{~V} \mathrm{~m}^{-2}$.
*Present address: Laboratory for Muon-Spin Spectroscopy, Paul Scherrer Institute, 5232 Villigen-PSI, Switzerland.

${ }^{1}$ J. S. Gardner, M. J. P. Gingras, and J. E. Greedan, Rev. Mod. Phys. 82, 53 (2010).

${ }^{2}$ M. J. Harris, S. T. Bramwell, D. F. McMorrow, T. Zeiske, and K. W. Godfrey, Phys. Rev. Lett. 79, 2554 (1997).

${ }^{3}$ A. P. Ramirez, A. Hayashi, R. J. Cava, R. Siddharthan, and B. S. Shastry, Nature (London) 399, 333 (1999).

${ }^{4}$ J. A. Hodges, P. Bonville, A. Forget, A. Yaouanc, P. Dalmas de Réotier, G. André, M. Rams, K. Królas, C. Ritter, P. C. M. Gubbens, C. T. Kaiser, P. J. C. King, and C. Baines, Phys. Rev. Lett. 88, 077204 (2002).

${ }^{5}$ I. Mirebeau, A. Apetrei, J. Rodríguez-Carvajal, P. Bonville,

A. Forget, D. Colson, V. Glazkov, J. P. Sanchez, O. Isnard, and E. Suard, Phys. Rev. Lett. 94, 246402 (2005).

${ }^{6}$ P. Dalmas de Réotier, A. Yaouanc, L. Keller, A. Cervellino, B. Roessli, C. Baines, A. Forget, C. Vaju, P. C. M. Gubbens, A. Amato, and P. J. C. King, Phys. Rev. Lett. 96, 127202 (2006).

${ }^{7}$ J. D. M. Champion, A. S. Wills, T. Fennell, S. T. Bramwell, J. S. Gardner, and M. A. Green, Phys. Rev. B 64, 140407 (2001).

${ }^{8}$ J. R. Stewart, G. Ehlers, A. S. Wills, S. T. Bramwell, and J. S. Gardner, J. Phys.: Condens. Matter 16, L321 (2004).

${ }^{9}$ A. S. Wills, M. E. Zhitomirsky, B. Canals, J. P. Sanchez, P. Bonville, P. Dalmas de Réotier, and A. Yaouanc, J. Phys.: Condens. Matter 18, L37 (2006).

${ }^{10}$ M. I. Brammall, A. K. R. Briffa, and M. W. Long, Phys. Rev. B 83, 054422 (2011).

${ }^{11}$ E. Bertin, P. Bonville, J.-P. Bouchaud, J. A. Hodges, J. P. Sanchez, and P. Vulliet, Eur. Phys. J. B 27, 347 (2002).

${ }^{12}$ A. Yaouanc, P. Dalmas de Réotier, V. Glazkov, C. Marin, P. Bonville, J. A. Hodges, P. C. M. Gubbens, S. Sakarya, and C. Baines, Phys. Rev. Lett. 95, 047203 (2005).

${ }^{13}$ Y. Chapuis, P. Dalmas de Réotier, C. Marin, A. Yaouanc, A. Forget, A. Amato, and C. Baines, Physica B 404, 686 (2009).

${ }^{14}$ J. A. Hodges, P. Bonville, A. Forget, M. Rams, K. Królas, and G. Dhalenne, J. Phys.: Condens. Matter 13, 9301 (2001).

${ }^{15}$ Y. Yasui, M. Soda, S. Iikubo, M. Ito, M. Sato, N. Hamaguchi, T. Matsushita, N. Wada, T. Takeuchi, N. Aso, and K. Kakurai, J. Phys. Soc. Jpn. 72, 3014 (2003).

${ }^{16}$ J. S. Gardner, G. Ehlers, N. Rosov, R. W. Erwin, and C. Petrovic, Phys. Rev. B 70, 180404(R) (2004).
${ }^{17}$ L.-J. Chang, S. Onoda, Y. Su, Y.-J. Kao, K.-D. Tsuei, Y. Yasui, K. Kakurai, and M. R. Lees, Nat. Commun. 3, 992 (2012).

${ }^{18}$ J. A. Hodges, P. Dalmas de Réotier, A. Yaouanc, P. C. M. Gubbens, P. J. C. King, and C. Baines, J. Phys.: Condens. Matter 23, 164217 (2011).

${ }^{19}$ J. D. M. Champion, M. J. Harris, P. C. W. Holdsworth, A. S. Wills, G. Balakrishnan, S. T. Bramwell, E. Čižmár, T. Fennell, J. S. Gardner, J. Lago, D. F. McMorrow, M. Orendáč, A. Orendáčová, D. M. McK. Paul, R. I. Smith, M. T. F. Telling, and A. Wildes, Phys. Rev. B 68, 020401 (2003).

${ }^{20}$ J. P. C. Ruff, J. P. Clancy, A. Bourque, M. A. White, M. Ramazanoglu, J. S. Gardner, Y. Qiu, J. R. D. Copley, M. B. Johnson, H. A. Dabkowska, and B. D. Gaulin, Phys. Rev. Lett. 101, 147205 (2008).

${ }^{21}$ A. K. R. Briffa, R. J. Mason, and M. W. Long, Phys. Rev. B 84, 094427 (2011).

${ }^{22}$ J. Villain, R. Bidaux, J.-P. Carton, and R. Conte, J. Phys. (France) 41, 1263 (1980)

${ }^{23}$ S. T. Bramwell, M. J. P. Gingras, and J. N. Reimers, J. Appl. Phys. 75, 5523 (1994).

${ }^{24}$ J. D. M. Champion and P. C. W. Holdsworth, J. Phys.: Condens. Matter 16, S665 (2004).

${ }^{25}$ M. E. Zhitomirsky, M. V. Gvozdikova, P. C. W. Holdsworth, and R. Moessner, Phys. Rev. Lett. 109, 077204 (2012).

${ }^{26}$ L. Savary, K. A. Ross, B. D. Gaulin, J. P. C. Ruff, and L. Balents, arXiv:1204.1320.

${ }^{27}$ K. B. Helean, S. V. Ushakov, C. E. Brown, A. Navrotsky, J. Lian, R. C. Ewing, J. M. Farmer, and L. A. Boatner, J. Solid State Chem. 177, 1858 (2004).

${ }^{28}$ A. Bertin, Y. Chapuis, P. Dalmas de Réotier, and A. Yaouanc, J. Phys.: Condens. Matter 24, 256003 (2012).

${ }^{29}$ H. W. J. Blöte, R. F. Wielinga, and W. J. Huiskamp, Physica 43, 549 (1969).

${ }^{30}$ S. T. Bramwell, M. N. Field, M. J. Harris, and I. P. Parkin, J. Phys.: Condens. Matter 12, 483 (2000).

${ }^{31}$ A. Poole, A. S. Wills, and E. Lelièvre-Berna, J. Phys.: Condens. Matter 19, 452201 (2007).

${ }^{32}$ In fact the authors of Refs. 19 and 31 label this representation as $\Gamma_{5}$. Our labeling is consistent with http://www.cryst.ehu.es/cgi-bin/ rep/programs/sam/point.py?sg=221. 
${ }^{33}$ H. B. Cao, I. Mirebeau, A. Gukasov, P. Bonville, and C. Decorse, Phys. Rev. B 82, 104431 (2010).

${ }^{34}$ S. S. Sosin, L. A. Prozorova, M. R. Lees, G. Balakrishnan, and O. A. Petrenko, Phys. Rev. B 82, 094428 (2010).

${ }^{35}$ A. Abragam and B. Bleaney, Electron Paramagnetic Resonance of Transition Ions (Clarendon, Oxford, 1970).

${ }^{36} \mathrm{P}$. Bonville, J. A. Hodges, P. Imbert, and F. Hartmann-Boutron, Phys. Rev. B 18, 2196 (1978).

${ }^{37}$ Y. Chapuis, A. Yaouanc, P. Dalmas de Réotier, S. Pouget, P. Fouquet, A. Cervellino, and A. Forget, J. Phys.: Condens. Matter 19, 446206 (2007).

${ }^{38}$ A. Yaouanc, P. Dalmas de Réotier, Y. Chapuis, C. Marin, S. Vanishri, D. Aoki, B. Fåk, L. P. Regnault, C. Buisson, A. Amato, C. Baines, and A. D. Hillier, Phys. Rev. B 84, 184403 (2011).

${ }^{39}$ A. P. Ramirez, in Handbook of Magnetic Materials, Vol. 13, edited by K. H. J. Buschow (Elsevier, 2001).

${ }^{40}$ R. Siddharthan, B. S. Shastry, A. P. Ramirez, A. Hayashi, R. J. Cava, and S. Rosenkranz, Phys. Rev. Lett. 83, 1854 (1999).

${ }^{41}$ R. K. Harris, E. D. Becker, S. M. Cabral de Menezes, R. Goodfellow, and P. Granger, Pure Appl. Chem. 73, 1795 (2001).

${ }^{42}$ W. Zheng, J. O. Fjærestad, R. R. P. Singh, R. H. McKenzie, and R. Coldea, Phys. Rev. Lett. 96, 057201 (2006).

${ }^{43} \mathrm{~S}$. W. Lovesey, Theory of Neutron Scattering from Condensed Matter, Vol. 2 (Clarendon, Oxford, 1986).

${ }^{44}$ D. H. Ryan, J. M. Cadogan, and R. Gagnon, Phys. Rev. B 68, 014413 (2003).

${ }^{45}$ P. Bonville, J. Phys.: Conf. Series 217, 012119 (2010).

${ }^{46}$ M. E. Fisher, J. Math. Phys. 5, 944 (1964).

${ }^{47}$ A. Kornblit and G. Ahlers, Phys. Rev. B 8, 5163 (1973).

${ }^{48}$ Y. Chapuis, A. Yaouanc, P. Dalmas de Réotier, C. Marin, S. Vanishri, S. H. Curnoe, C. Vâju, and A. Forget, Phys. Rev. B 82, 100402(R) (2010).

${ }^{49}$ A. Yaouanc and P. Dalmas de Réotier, Muon Spin Rotation, Relaxation, and Resonance: Applications to Condensed Matter, International Series of Monographs on Physics 147 (Oxford University Press, Oxford, 2011).

${ }^{50}$ J. Lago, T. Lancaster, S. J. Blundell, S. T. Bramwell, F. L. Pratt, M. Shirai, and C. Baines, J. Phys.: Condens. Matter 17, 979 (2005).
${ }^{51}$ For instance the spectrum measured at $21 \mathrm{mK}$ under a field of $1.3 \mathrm{~T}$ can nicely be fitted to a stretched exponential relaxation function $a_{0} \exp \left[-\left(\lambda_{Z} t\right)^{\beta}\right]$ with $\beta=0.86(3)$.

${ }^{52}$ A. G. Redfield, IBM J. Research and Development 1, 19 (1957).

${ }^{53}$ K. C. Rule, G. Ehlers, J. S. Gardner, Y. Qiu, E. Moskvin, K. Kiefer, and S. Gerischer, J. Phys.: Condens. Matter 21, 486005 (2009).

${ }^{54} \mathrm{The}$ form factor of the $\mathrm{Er}^{3+}$ ion was taken from http://www.neutron.ethz.ch/research/resources/formfactor.

${ }^{55}$ S.-H. Lee, C. Broholm, W. Ratcliff, G. Gasparovic, Q. Huang, T. H. Kim, and S.-W. Cheong, Nature (London) 418, 856 (2002).

${ }^{56}$ T. Yavors'kii, T. Fennell, M. J. P. Gingras, and S. T. Bramwell, Phys. Rev. Lett. 101, 037204 (2008).

${ }^{57}$ P. H. Conlon and J. T. Chalker, Phys. Rev. B 81, 224413 (2010).

${ }^{58}$ K. A. Ross, L. Savary, B. D. Gaulin, and L. Balents, Phys. Rev. X 1, 021002 (2011).

${ }^{59}$ O. A. Starykh, A. V. Chubukov, and A. G. Abanov, Phys. Rev. B 74, R180403 (2006).

${ }^{60}$ A. L. Chernyshev and M. E. Zhitomirsky, Phys. Rev. Lett. 97, 207202 (2006).

${ }^{61}$ S. H. Curnoe, Phys. Rev. B 75, 212404 (2007).

${ }^{62}$ S. H. Curnoe, Phys. Rev. B 78, 094418 (2008).

${ }^{63}$ P. McClarty, S. Curnoe, and M. Gingras, J. Phys.: Conf. Series 145, 012032 (2009).

${ }^{64}$ H. R. Molavian, M. J. P. Gingras, and B. Canals, Phys. Rev. Lett. 98, 157204 (2007).

${ }^{65}$ L. Savary and L. Balents, Phys. Rev. Lett. 108, 037202 (2012).

${ }^{66}$ S. Onoda and Y. Tanaka, Phys. Rev. Lett. 105, 047201 (2010).

${ }^{67}$ S. Onoda, J. Phys.: Conf. Series 320, 012065 (2011).

${ }^{68}$ S. Onoda and Y. Tanaka, Phys. Rev. B 83, 094411 (2011).

${ }^{69}$ B. Z. Malkin, T. T. A. Lummen, P. H. M. Loosdrecht, G. Dhalenne, and A. R. Zakirov, J. Phys.: Condens. Matter 22, 276003 (2010).

${ }^{70}$ J. D. Thompson, P. A. McClarty, H. M. Rønnow, L. P. Regnault, A. Sorge, and M. J. P. Gingras, Phys. Rev. Lett. 106, 187202 (2011).

${ }^{71}$ J. Pelzl, S. Hüfner, and S. Scheller, Z. Phys. 231, 377 (1970).

${ }^{72}$ A. J. Freeman and J. P. Desclaux, J. Magn. Magn. Mater. 12, 11 (1979). 